\title{
Changeover Formulations for Discrete-Time Mixed-integer Programming Scheduling Models
}

\author{
Sara Velez, Yachao Dong and Christos T. Maravelias* \\ Department of Chemical and Biological Engineering, University of Wisconsin - Madison \\ 1415 Engineering Dr., Madison, WI 53706, USA
}

\begin{abstract}
Changeover times can have a significant impact on the scheduling of manufacturing operations. Unfortunately, accounting for changeovers in mixed-integer programming (MIP) scheduling formulations makes the resulting models computationally more expensive. We propose five new formulations for sequence-dependent changeovers, applicable to a wide range of scheduling problems. We generate constraints for different sets of time points and sets of tasks. We also propose valid inequalities for makespan minimization. Furthermore, we prove results regarding the relative tightness of each formulation. Finally, we perform a computational study. Interestingly, we find that tighter formulations do not always lead to faster solution times, and we show that some of the new formulations perform better than the previously proposed ones.
\end{abstract}

\section{Keywords:}

Scheduling; integer programming; formulation tightness; manufacturing scheduling.

*Corresponding author: Christos T. Maravelias

Tel.: +1 6082659026

Fax: +1 6082625434

Email address: maravelias@wisc.edu

(C) 2017. This manuscript version is made available under the Elsevier user license 


\section{Introduction}

A wide range of mixed-integer programming (MIP) models (Wolsey, 1998) have been proposed in the literature to address manufacturing scheduling problems, and chemical production scheduling problems in particular (Méndez et al., 2006; Maravelias, 2012; Harjunkoski et al., 2014). One of the differentiating attributes of the models is the modeling of time. In discrete-time models, the scheduling horizon $\eta$ is divided into $T$ periods of fixed length $\delta=\eta / T$, defining $T+1$ time points (i.e., period t starts at time point $t-1$ and ends at time point $\mathrm{t}$ ). In continuous-time models, the horizon is divided into a known number of periods with variable length. Discrete-time models have several advantages over their continuous-time counterparts: they (1) are tighter (Sundaramoorthy and Maravelias, 2011a; Velez and Maravelias, 2013), (2) can easily handle intermediate release and due times, (3) can model holding and backlogging costs linearly, and (4) can be readily extended to handle events during the execution of a task. Furthermore, computational study showed that discrete-time models for problems in network production environments (i.e., environments where tasks produce and consume multiple materials and batches of materials can be mixed and split) can often be solved faster and find better solutions compared to continuous-time models (Sundaramoorthy and Maravelias, 2011a). However, because the size of discrete-time models grows at least linearly with the number of periods, the disadvantage of discrete-time models is the large number of binary variables and constraints, especially when sequence-dependent changeovers are considered. Such changeovers are common in the process industries (e.g., commodity, specialty, and fine chemicals; food and beverage manufacturing; pharmaceutical manufacturing; consumer goods), where cleaning-in-place, sterilization-in-place, maintenance, material transfer, and unit setup activities need to be performed between different tasks. Accordingly, the goal of this paper is to study discrete-time formulations for the modeling of sequence-dependent changeovers.

Solution methods for discrete-time MIP models for single-machine and parallel-machine problems include cutting planes (Wolsey, 1990; Sousa and Wolsey, 1992; van den Akker et al., 1999), column generation (van den Akker et al., 2000), branch-and-cut and column generation (Sadykov and Wolsey, 2006), as well as hybrid decomposition methods (Jain and Grossmann, 2001; Maravelias, 2006; Hooker, 2007b; Coban and Hooker, 2013). Wolsey has presented a survey of methods to improve changeover formulations for production planning and scheduling problems, which covers lot-sizing, single item problems, and machine-sequencing problems, and discusses time-indexed formulations (Wolsey, 1997). In terms of continuous-time models, a reformulation of the makespan minimization problem (Avalos-Rosales et al., 2013), a Benders decomposition (Tran and Beck, 2012), and a MIP-based decomposition (Kopanos et al., 2010) have been proposed.

If changeovers do not require resources and do not incur a cost, then changeover times can be enforced by simply allowing enough idle time between tasks (Kondili et al., 1993; Shah et al., 1993; Wolsey, 1997, Moniz et al., 2013). However, if resources are needed or costs need to be modeled, additional binary variables are necessary (Karmarkar and Schrage, 1985; Sahinidis and Grossmann, 
1991; Kondili et al., 1993; Zentner et al., 1994; Kelly and Zyngier, 2007). In this paper, we study changeovers for processes that neither require resources nor incur a cost.

In Section 2, we introduce the processes that we are interested in, describe their corresponding MIP models, and present three changeover formulations from the literature (Kondili et al., 1993; Shah et al., 1993; Wolsey, 1997). In Section 3, we present five new formulations; in Section 4, we present remarks on (1) tightening of the proposed formulations, (2) alternative formulations when some standard assumptions are relaxed, and (3) a valid inequality for makespan minimization. Section 5 presents results regarding the relative tightness of the formulations. In Section 6, we present computational results. We use lowercase italic letters for indices, uppercase italic letters for variables, uppercase bold letters for sets, and lowercase Greek letters for parameters.

\section{Background}

We consider three variations on the single-stage environment with: (1) a single unit (machine), (2) parallel units, and (3) parallel units with unequal capacities. The horizon, $\eta$, is divided into $\mathrm{T}$ uniform intervals of length $\delta=\eta / T$, with $T+1$ time points $t \in\{0,1, \ldots, T\}$ occurring at $t \delta$. We use these three problems to study our new formulations because of their simplicity and because they represent three general classes of problems (see discussion in §2.5). We emphasize that the changeover constraints developed here can be readily used in any discrete-time model.

\subsection{Single Unit}

The single-unit problem consists of a set of tasks (jobs), $i \in \mathbf{I}$, with a fixed processing time, $\tau_{i} \delta$. We include a binary variable: $X_{i t}=1$ if and only if task $i$ starts at time point t. We assume that each task must be run exactly once (constraints (1)) and only one task can run at a time (constraints (2)).

$$
\begin{gathered}
\sum_{t} X_{i t}=1 \quad \forall i \\
\sum_{i} \sum_{t^{\prime}=t-\tau_{i}+1}^{t} X_{i t^{\prime}} \leq 1 \quad \forall t
\end{gathered}
$$

We can minimize makespan, $M S \in \mathbb{R}^{+}$,

$$
M S \geq \sum_{i}\left(t+\tau_{i}\right) X_{i t} \quad \forall t
$$

We can also minimize tardiness, $T R D \in \mathbb{R}^{+}$,

$$
T R D=\sum_{i} T R D_{i}
$$

where $T R D_{i} \in \mathbb{R}^{+}$denotes the tardiness of task i. $T R D_{i}$ is defined in constraints (5), where $\phi_{i}$ is the due time of taski: 


$$
T R D_{i} \geq \sum_{t=\phi_{i}-\tau_{i}+1}^{T}\left(t-\phi_{i}+\tau_{i}\right) X_{i t} \quad \forall i
$$

\subsection{Parallel Units}

When there are parallel units (machines), we introduce a new index, $j \in \mathbf{J}$, for units; the processing times, $\tau_{i j}$, are unit-dependent. To generate a schedule we must assign each task to a unit, so the binary variables are indexed by $\mathrm{j}: X_{i j t}=1$ if and only if task $i$ starts in unit $\mathrm{j}$ at time point t. We still assume that each task must be run exactly once (constraints (6), where $\mathbf{J}_{i}$ is the set of units that can process task $i$ ), and only one task can run at a time on a unit (constraints (7)).

$$
\begin{gathered}
\sum_{j \in \mathbf{J}_{i}, t} X_{i j t}=1 \quad \forall i \\
\sum_{i} \sum_{t^{\prime}=t-\tau_{i j}+1}^{t} X_{i j t^{\prime}} \leq 1 \quad \forall j, t
\end{gathered}
$$

Again, we consider makespan minimization,

$$
M S \geq \sum_{i}\left(t+\tau_{i j}\right) X_{i j t} \quad \forall j, t
$$

Since a task may run on different units and the cost may be different in each unit, we also consider cost, $C S T \in \mathbb{R}^{+}$, minimization, where $\alpha_{i j}$ is the cost to run task $i$ in unit $\mathrm{j}$.

$$
\text { CST }=\sum_{i, j, t} \alpha_{i j} X_{i j t}
$$

For cost minimization, we consider the case where each task i has a hard due date (deadline), $\bar{\phi}_{i}$,

$$
X_{i j t}=0 \quad \forall i, j, t>\bar{\phi}_{i}-\tau_{i}
$$

We also consider tardiness minimization, $T R D$, with constraints (4) and (11)

$$
T R D_{i} \geq \sum_{j} \sum_{t=\phi_{i}-\tau_{i j}+1}^{T}\left(t-\phi_{i}+\tau_{i j}\right) X_{i j t} \quad \forall i
$$

\subsection{Parallel Units with Unequal Capacities}

Each unit $\mathrm{j}$ has capacity $\beta_{j}$ and (the output of) each task $i$ has known demand, $\xi_{i}$. When units have unequal capacities, we cannot calculate how many times each task must run prior to optimization, so we replace constraints (6) with,

$$
\sum_{j \in \mathbf{J}_{i}, t} \beta_{j} X_{i j t} \geq \xi_{i} \quad \forall i
$$


Constraints (7) are also included to ensure that each unit processes at most one task at a time. Again, we consider MS, CST, and TRD minimization. The constraints for different problems and objective functions are summarized in Table 1.

Table 1. Constraints used for different production environments and objective functions.

\begin{tabular}{cccc}
\hline Production environments & $\min$ MS & $\min$ TRD & $\min$ CST \\
\hline Single unit & $(1)(2)(3)$ & $(1)(2)(4)(5)$ & \\
Parallel units & $(6)(7)(8)$ & $(4)(6)(7)(11)$ & (6) (7) (9) (10) \\
Parallel units with unequal capacities & $(7)(8)(12)$ & $(4)(7)(11)(12)$ & $(7)(9)(10)(12)$ \\
\hline
\end{tabular}

\subsection{Assumptions and Literature Formulations}

The changeover time after task $i$ finishes and before task $i^{\prime}$ starts on unit $\mathrm{j}$ is denoted by $\sigma_{i i^{\prime}}{ }^{\prime}$ in terms of the number of periods.

Assumptions. We make three assumptions.

Assumption 1. The changeover time between task $i$ and task $i^{\prime}$ is less than both $\tau_{i j}$ and $\tau_{i^{\prime} j}$ $\left(\sigma_{i i}{ }^{\prime} j<\min \left\{\tau_{i j}, \tau_{i^{\prime} j}\right\}\right)$.

Assumption 2. Changeover times satisfy the triangle inequality $\left(\sigma_{i i^{\prime \prime} j}<\sigma_{i i_{j}{ }^{\prime}}+\tau_{i^{\prime} j}+\sigma_{i^{\prime} i^{\prime \prime} j}\right)$.

Assumption 3. Tasks do not have a changeover with themselves $\left(\sigma_{i i j}=0\right)$.

For the single-unit problem, one drops the index $\mathrm{j}$ in $\tau_{i j}$ and $\sigma_{i i}{ }^{\prime}{ }$, and the assumptions are then analogous to those for the parallel-units problem. The changeover constraints presented in the remaining of the article can be written for the single-unit problem, simply by replacing variables $X_{i j t}$ by variables $X_{i t}$. Also, when processing and/or changeover times are unit independent, all the constraints in the paper still apply.

Formulations. Three discrete-time formulations have appeared in the literature to enforce changeover times without additional binary variables.

Constraints (K). Kondili et al. (Kondili et al., 1993) used a big-M constraint:

$$
\sum_{i \neq i} \sum_{t^{\prime}=t+\tau_{i j}}^{t+\tau_{i j}+\sigma_{i i^{\prime} j}-1} X_{i^{\prime} j t^{\prime}} \leq M\left(1-X_{i j t}\right) \quad \forall i, j, t
$$

Although no value for $\mathrm{M}$ was suggested, one that is obviously large enough is

$$
M_{i j}=\sum_{i \neq i} \sum_{t^{\prime}=t+\tau_{i j}}^{t+\tau_{i j}+\sigma_{i i^{\prime}{ }^{\prime}}-1} 1=\sum_{i \neq i} \sigma_{i i^{\prime} j}
$$

While smaller values of $\mathrm{M}$ can be found, we observed that the value of $\mathrm{M}$ does not make a significant difference in the solution time (see Supplementary Material).

Constraints (SH). Shah et al. (Shah et al., 1993) eliminated the big-M constraint by considering pair of tasks: 


$$
X_{i j t}+X_{i^{\prime} j t^{\prime}} \leq 1 \quad \forall i, i^{\prime} \neq i, j, t, t-\tau_{i^{\prime} j}-\sigma_{i^{\prime}}{ }_{i j}<t^{\prime} \leq t-\tau_{i^{\prime} j}
$$

Constraints (W). Finally, Wolsey (Wolsey, 1997) proposed the following constraints,

$$
\sum_{t^{\prime}=t-\tau_{i j}-\sigma_{i i^{\prime} j^{\prime}}+1}^{t} X_{i j t^{\prime}}+\sum_{t^{\prime}=t-\tau_{i^{\prime} j}-\sigma_{i^{\prime} i j}+1}^{t} X_{i^{\prime} j t^{\prime}} \leq 1 \quad \forall i, i^{\prime} \neq i, j, t
$$

Constraints (16) include the binary variable for task $i$ for more than $\tau_{i j}$ consecutive time points, which prevents task $i$ from occurring back-to-back, so they are valid only when each task is restricted to run once. In the paper, we also consider problems where multiple executions of the same task are allowed (in problems of parallel units with unequal capacities).

\subsection{Remarks}

The problem in $\S 2.1$ is a traditional scheduling problem which involves only sequencing and timing decisions. In addition to sequencing and timing, the problem in $\$ 2.2$ includes also task-unit assignment decisions, and the problem in $\$ 2.3$ includes also batching decisions. For the problems in $\S 2.1$ and $\S 2.2$, each task is to be scheduled once, whereas in the problem of $\S 2.3$, the number of times that a task is scheduled is determined by the optimization model. Discrete-time MIP models can be applied to all of the aforementioned problem classes, while other methods are limited in the types of problems they can address or the objectives they can handle. For example, while constraint programming (CP) and hybrid MIP/CP methods have been shown to be effective for various classes of problems (Jain and Grossmann, 2001; Maravelias and Grossmann, 2004; Sadykov and Wolsey, 2006; Hooker, 2007a; Zeballos et al., 2011; Novara et al., 2016), there are no CP and hybrid MIP/CP methods readily applicable to wide range of problems with batching decisions. Similarly, combinatorial branch-and-bound algorithms have not been developed for problems that include task-unit assignments, and the developed algorithms are written for specific objectives (Brucker and Thiele, 1996; Baptiste et al., 2003; Artigues and Feillet, 2008; Chandra et al., 2014). Also, with the exception of MIP-based approaches, most existing methods cannot handle problems with variable batch sizes and thus variable number of tasks.

Furthermore, discrete-time models can be easily extended to account for time-varying resource availability and events during the execution of a task (e.g., intermediate material loading), and can be used as a basis for many problems. For example, they can be extended to address problems in production environments where batches can be split apart or mixed (Sundaramoorthy and Maravelias, 2011b), problems with other types of constraints (e.g., limited utilities) (Zyngier and Kelly, 2009; Velez and Maravelias, 2013), as well as problems with various objective functions (Merchan et al., 2016). Thus, our choice to focus on discrete-time MIP formulations means that the proposed changeover constraints will be applicable to a wide range of problems.

\section{Proposed Formulations}

In this section, we present 5 new changeover formulations, classified based on: 
(1) the number of tasks for which each changeover constraint is written: a single task (S) or a pair of tasks $(\mathrm{P})$;

(2) the number of time points for which each changeover constraint is written: all pairs of time points within an interval depending on the processing and changeover times (I), a subset of pairs of time points (II), or a single time point (III).

For instance, the three formulations presented in $\$ 2.4$ can be classified accordingly: constraints (K) are type (S)/(III); constraints (SH) are type (P)/(I); constraints (W) are type (P)/(III). The 5 new formulations we present in §3.1-3.5 are named based on their classification: (SI), (SII), (SIIT) (a tighter version of $(\mathrm{SII}))$, (SIII), and $(\mathrm{P})=(\mathrm{PI})=(\mathrm{PII})=(\mathrm{PIII})$.

To describe these formulations, we introduce the following sets,

(1) $\mathbf{T}_{i j t}^{P}=\left\{t^{\prime} \mid t-\tau_{i j}+1 \leq t^{\prime} \leq t\right\}$, referred to as the set of processing time points for $\mathrm{i}, \mathrm{j}$, $\mathrm{t}$.

(2) $\mathbf{T}_{i^{\prime} i j t}^{C}=\left\{t^{\prime} \mid t-\tau_{i^{\prime} j}-\sigma_{i^{\prime} i j}+1 \leq t^{\prime} \leq t-\tau_{i^{\prime}}{ }^{\prime}\right\}$, referred to as the set of changeover time points from $i^{\prime}$ to $i$ for $\mathrm{j}$, t.

Considering a given task $\mathrm{i}$, time point $\mathrm{t}$, and unit $\mathrm{j}$, constraints (7) enforce $X_{i^{\prime}} j t^{\prime}=0$, for each $i^{\prime} \in \mathbf{I}$, $t^{\prime} \in \mathbf{T}_{i^{\prime} j t}^{P}$, if $X_{i j t}=1$; while changeover constraints should enforce $X_{i^{\prime} j t^{\prime}}=0$, for $i^{\prime} \in \mathbf{I} \backslash\{i\}, t^{\prime} \in \mathbf{T}_{i^{\prime} i j t}^{C}$, if $X_{i j t}=1$. Thus, variables $X_{i^{\prime} j t^{\prime}}$ with $t^{\prime} \in \mathbf{T}_{i^{\prime}}^{C}{ }_{i j t}$ should be included in at least one changeover constraint. Including $X_{i^{\prime}}{ }^{\prime} t^{\prime}$ with $t^{\prime} \in \mathbf{T}_{i^{\prime} j t}^{P^{\prime}}$ may tighten the changeover constraints, but it is not necessary. In fact, we found that including $X_{i^{\prime} j t^{\prime}}$ with $t^{\prime} \in \mathbf{T}_{i^{\prime} j t}^{P^{\prime}}$ resulted in longer solution times even though the resulting changeover constraints are tighter and the number of these constraints remains the same. This computational observation motivates us to exclude $X_{i^{\prime}}{ }^{\prime} t^{\prime}$ with index $t^{\prime} \in \mathbf{T}_{i^{\prime} j t}^{P}$, when formulating most of the changeover constraints (except (SIIT)).

To illustrate the ideas behind the derivation of the proposed constraints we will use graphs that combine the Gantt chart that corresponds to a specific solution of the MIP model and the binary variables, $X_{i j t}$, that are used to generate the Gantt chart. For example, in Figure 1, the execution of task T3 from $t=14$ to $t=22$ (represented by a block) is the result of binary variable $X_{\mathrm{T} 3, j, 14}$ being equal to 1 . In this graph, binary variables $X_{i j t}$ will be represented by points whose $\mathrm{x}$ coordinate indicate the time index $\mathrm{t}$ and their y coordinate indicates task $i$. Furthermore, processing time points $\left(t^{\prime} \in \mathbf{T}_{i^{\prime} j t}^{P}\right)$ are shown as squares, while changeover time points $\left(t^{\prime} \in \mathbf{T}_{i^{\prime} i j t}^{C}\right)$ are shown as circles. In Figures 1-3, 6, 7, we illustrate the logic behind the various constraints using the data in Table 2 and considering $i=\mathrm{T} 3, t=14$. In these figures, we will not use index $\mathrm{j}$ because all types of changeover constraints are written for a specific unit.

Table 2. Data used in examples (with $v_{\text {in }}$ defined in $\S 3.2$ ).

\begin{tabular}{|c|c|c|c|c|c|c|}
\hline & \multirow[b]{2}{*}{$\tau_{\mathrm{i}}$} & \multicolumn{3}{|c|}{$\sigma_{i^{\prime} i}\left(i^{\prime}=\right.$ left, $\mathrm{i}=$ top $)$} & \multicolumn{2}{|c|}{$v_{\text {in }}$} \\
\hline & & $\mathrm{T} 1$ & $\mathrm{~T} 2$ & T3 & $\mathrm{n}=1$ & $\mathrm{n}=2$ \\
\hline T1 & 7 & 0 & 1 & 6 & 4 & -- \\
\hline T2 & 4 & 1 & 0 & 3 & 7 & -- \\
\hline T3 & 8 & 4 & 2 & 0 & 4 & 11 \\
\hline
\end{tabular}




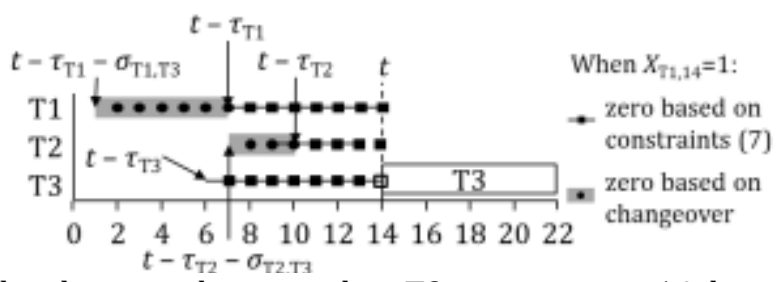

Figure 1. Binary variables that must be zero when T3 starts at time 14, based on the data of Table 2 .

\subsection{Constraints (SI)}

Constraints (SI) are written for a single task and unit and for pairs of time points, as follows:

$$
\begin{aligned}
& \left.\left.X_{i j t}+\sum_{i \neq i} \sum_{t^{\prime \prime}=\max }^{\min \left\{t^{\prime}, t-\sigma_{i^{\prime}}{ }^{\prime}, t-\tau_{i^{\prime}}\right\}}\right\}-\tau_{i^{\prime} j^{\prime}+1}\right\} X_{i^{\prime} j t^{\prime \prime}} \leq 1 \\
& \forall i, j, t, t-\max \left\{\min _{i \neq i} \tau_{i^{\prime} j}, \max _{i \neq i} \sigma_{i^{\prime} i j}\right\} \leq t^{\prime} \leq t-\min _{i \neq i} \tau_{i^{\prime} j}
\end{aligned}
$$

Considering a given task $\mathrm{i}$, time point $\mathrm{t}$, and unit $\mathrm{j}$, the bounds on $t^{\prime}$ and $t^{\prime \prime}$ are explained in the following 2 paragraphs. We define $t^{\prime}$ to be the latest time point for which any $X_{i^{\prime}}{ }^{\prime \prime} t^{\prime \prime}$ for task $i^{\prime} \neq i$ can be included in the constraint.

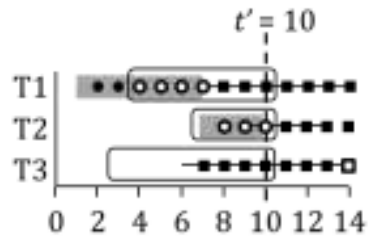

(a) $t^{\prime}=10$

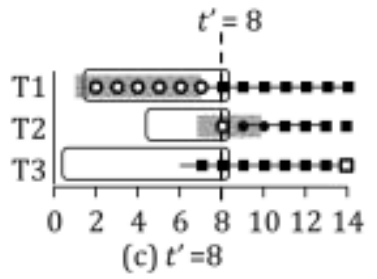

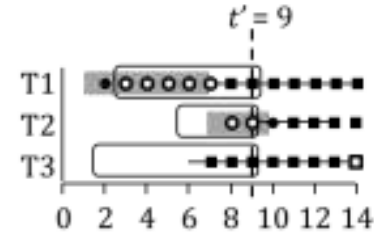

(b) $t^{\prime}=9$

* Changeover $\left(\sigma_{i}\right)$

- Processing time $\left(\tau_{i}\right)$ ao Points included in (SI)

Points included in constraints (7) at $t^{\prime}$

Figure 2. Constraints (SI) for $i=\mathrm{T} 3, t=14$, and $t^{\prime}=8-10$, based on the data of Table 2 .

First, we set the limits on $t^{\prime \prime}$ for included variables $X_{i^{\prime}}{ }^{\prime} t^{\prime \prime}$. For each task $i^{\prime}$, we require that $t^{\prime \prime} \leq t^{\prime}$ based on our definition of $t^{\prime}$. The smallest time index $t^{\prime \prime}$ of included variables $X_{i^{\prime} j t^{\prime \prime}}$ cannot be smaller than $t^{\prime}-\tau_{i^{\prime}}{ }_{j}+1$. Including $X_{i^{\prime}}{ }_{j t}{ }^{\prime \prime}$ for at most $\tau_{i^{\prime}}{ }_{j}$ time points for any task $i^{\prime}$ ensures that the summation is less than one when $X_{i j t}=0$. If $t^{\prime}-\tau_{i^{\prime} j}+1 \leq t^{\prime \prime} \leq t^{\prime}$ is required, then we may include some variables $X_{i^{\prime} j t} t^{\prime \prime}$ with $t^{\prime \prime} \in \mathbf{T}_{i^{\prime} j t}^{P}$. To avoid this, we do not include $X_{i^{\prime} j t} t^{\prime \prime}$ for time points $t^{\prime \prime}$ earlier than $t-\tau_{i^{\prime} j}-\sigma_{i^{\prime} i j}+1$ or later than $t-\tau_{i^{\prime} j}$.

Next, we need to choose the bounds on $t^{\prime}$. The latest time point of $X_{i^{\prime}}{ }^{\prime} t^{\prime \prime}$ that must be included (that is, the latest of the round points in Figure 1) is $t-\min _{i^{\prime} \neq i}\left\{\tau_{i^{\prime} j}\right\}$, so we use this value as the upper bound on $t^{\prime}$. The earliest time point of $X_{i^{\prime}}{ }^{\prime} t^{\prime \prime}$ that must be included in some changeover constraints 
(that is, the earliest of the circles in Figure 1) is $t-\max _{i^{\prime} \neq i}\left\{\tau_{i^{\prime}{ }_{j}}+\sigma_{i^{\prime}}{ }_{i j}\right\}+1$. Since the summation over $t^{\prime \prime}$ includes up to $\tau_{i^{\prime} j}$ time points for a task $i^{\prime}$, the constraint for $t^{\prime}=t-\max _{i^{\prime} \neq i}\left\{\tau_{i^{\prime} j}-\tau_{i^{\prime} j}+\right.$ $\left.\sigma_{i^{\prime}}{ }_{i j}\right\}=t-\max _{i^{\prime} \neq i}\left\{\sigma_{i^{\prime}}{ }_{i j}\right\}$ is the earliest $t^{\prime}$ for which we need to write constraints (SI). To ensure the constraint is written for at least one $t^{\prime}$, we set the lower bound on $t^{\prime}$ to $t-\max \left\{\min _{i^{\prime} \neq i} \tau_{i^{\prime} j}, \max _{i^{\prime} \neq i} \sigma_{i}{ }^{\prime} i j\right\}$. Otherwise, simply choosing $t-\max _{i^{\prime} \neq i} \sigma_{i^{\prime} i j} \leq t^{\prime} \leq t-$ $\min _{i^{\prime} \neq i} \tau_{i^{\prime} j}$ will exclude all $t^{\prime}$ when the maximum changeover is less than the minimum processing time.

The correctness of (SI) is proved in Appendix 1. The correctness of all other proposed constraints can be proved in a similar way.

\subsection{Constraints (SII)}

Considering a given task $\mathrm{i}$, time point $\mathrm{t}$, and unit $\mathrm{j}$, constraints (SI) are written for more time points, $t^{\prime}$, than what is required to enforce changeovers. For example, constraints (SI) at $t^{\prime}=10$ and $t^{\prime}=8$ in Figure 2 are sufficient to enforce changeovers. Therefore, constraints (SII) are identical to (SI), but are written for a subset of pairs of time points. We use integer parameter $v_{i j n}$ to select the time points, $t^{\prime}=t-v_{i j n}$, for which we express (SI), where $\mathrm{n}$ indexes constraints (SII) and $\mathbf{N}_{i j}$ is the set of indices $\mathrm{n}$ for which $v_{i j n}$ is defined:

$$
X_{i j t}+\sum_{i \neq i} \sum_{t^{\prime \prime}=\max }^{\min \left\{t-v_{i j n}, t-\tau_{i^{\prime} j}\right\}} X_{\left.i^{\prime} j t^{\prime \prime}, t-\sigma_{i^{\prime}}{ }^{\prime}\right\}} \leq 1-\tau_{i^{\prime} j^{\prime}+1} \quad \forall i, j, t, n \in \mathbf{N}_{i j}
$$

We start by selecting $v_{i j 1}$ so that $t-v_{i j 1}$ is the latest time point $t^{\prime \prime}$ of $X_{i^{\prime} j t} t^{\prime \prime}$ that we want to include in the changeover constraint for any task $i^{\prime}$, i.e., the largest $t^{\prime \prime} \in \mathbf{T}_{i^{\prime} i j t}^{C}$. Therefore,

$$
v_{i j 1}=\min _{i \neq i} \tau_{i^{\prime} j}
$$

Note that $t-v_{i j 1}$ has the same value as the latest $t^{\prime}$ in constraints (SI). To find the values of the remaining $v_{i j n}$, we assume that we have values of $v_{i j n}$ for all $n \leq n^{*}$ and try to find the value of $v_{i j, n^{*}+1}$. We want to choose the value of $v_{i j, n *+1}$ to satisfy two conditions: (1) For any task $i^{\prime}$, all variables $X_{i}{ }^{\prime}{ }^{\prime \prime} t^{\prime \prime}$ during the changeover after time point $t-\tau_{i^{\prime}{ }_{j}}-v_{i j, n^{*}+1}$ must be included in at least one constraint whose index $\mathrm{n}$ is less than $n^{*}+1$, and (2) $v_{i j, n *+1}$ should be as large as possible without violating the first condition. The first condition ensures that for a given $i^{\prime}$, binary variables corresponding to changeover time points after $t-\tau_{i}{ }^{\prime} j-v_{i j, n^{*}+1}$ appear in constraints for smaller $\mathrm{n}$. The second condition ensures that we find the minimum number of constraints for (SII). For a task $i^{\prime}$ with $\sigma_{i^{\prime} i j} \leq v_{i j n^{*}}$, the constraint for $n \leq n^{*}$ includes all $X_{i^{\prime}}{ }^{\prime} t^{\prime \prime}$ with the time points after

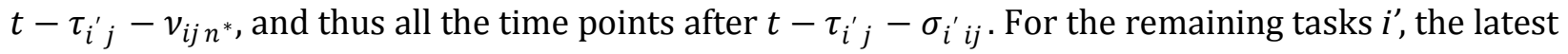
time point of $X_{i^{\prime}}{ }^{\prime \prime} t^{\prime \prime}$ that is not included in any constraint with $n \leq n^{*}$ is $t-\tau_{i^{\prime} j}-v_{i j n^{*}}$. We set the next value of $v_{i j, n^{*}+1}$ to the minimum of $\tau_{i^{\prime} j}+v_{i j n^{*}}$ over $i^{\prime}$ so that $t-v_{i j, n^{*}+1} \geq t-\tau_{i^{\prime} j}-v_{i j n^{*}}$ is the latest time point that does not appear in any constraint with $n \leq n^{*}$. The general equation to find the value of $v_{i j, n+1}$ is 


$$
v_{i j, n+1}=v_{i j n}+\min _{i^{\prime}: v_{i j, n}<\sigma_{i^{\prime} i j}} \tau_{i^{\prime} j}
$$

The largest index $\mathrm{n}$ for an $(i, j)$ pair satisfies that $v_{i j n}$ is greater than or equal to the changeover from any task to $i$, i.e., $v_{i j,\left|\mathbf{N}_{i j}\right|} \geq \max _{i{ }^{\prime} \neq i} \sigma_{i{ }^{\prime}}{ }_{i j}$. Figure 3 shows constraints (SII) for $i=\mathrm{T} 3, t=14$, and the calculated parameter $v_{i j n}$ can be found in Table 2 .

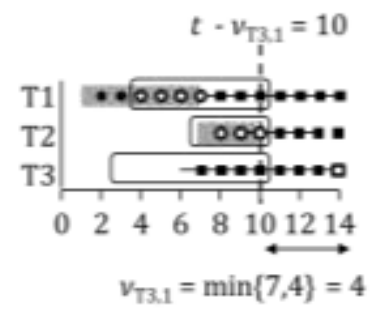

(a) $n=1$

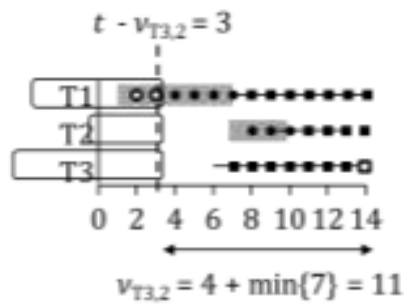

(b) $n=2$

Figure 3. Constraints (SII) for $i=\mathrm{T} 3, t=14$, and $n=1$ and 2 , based on the data of Table 2 .

Note. The hollow points represent binary variables included in each constraint. In (a), $v_{\mathrm{T} 3,1}$ is the difference of $\mathrm{t}$ from the latest hollow circle. In (b), $v_{\mathrm{T} 3,2}$ is as large as possible without excluding any circles that are not hollow for $n=1$. The constraint for $n=1$ is the same as (SI) for $t^{\prime}=10$. Comparing (b) with Figure 2c shows that the constraint for $n=2$ is dominated by constraint (SI) at $t^{\prime}=8$.

\subsection{Facet-defining Constraints (SIIT)}

Two modifications can be made to constraints (17). First, many of the inequalities (17) are redundant, as $t^{\prime}$ is written for each time point within $\left\{t-\max \left(\min _{i^{\prime} \neq i} \tau_{i^{\prime} j}, \max _{i^{\prime} \neq i} \sigma_{i^{\prime} i j}\right), \ldots, t-\right.$ $\left.\min _{i^{\prime} \neq i} \tau_{i^{\prime} j}\right\}$. Second, for the remaining necessary constraints, more variables related to $i$ and $i^{\prime}$ can be added to the left hand side (LHS) so that the constraints are tightened. Based on these two observations, we propose constraints (SIIT), which do not include redundant constraints, while making each constraint as tight as possible. The general form is,

$$
\sum_{t^{\prime}=t-\tau_{i j}+1}^{t} X_{i j t^{\prime}}+\sum_{i \neq i} \sum_{t^{\prime}=t-\omega a_{i j n} i^{\prime}}^{t-\omega b_{i j n i^{\prime}}} X_{i^{\prime} j t^{\prime}} \leq 1 \quad \forall i, j, t, n \in \mathbf{N}_{i j}
$$

where the first summation includes all variables corresponding to processing time points $\left(t^{\prime} \in \mathbf{T}_{i j t}^{P}\right)$ for task $i$, and the second term is a summation over $i^{\prime}$ and $t^{\prime} \in\left\{t-\omega a_{i j n} i^{\prime}, \ldots, t-\omega b_{i j n i^{\prime}}\right\}$ (parameters $\omega a_{i j n i}$ and $\omega b_{i j n i}$ will be defined later). Similar to (SII), we introduce $\left|\mathbf{N}_{i j}\right|$ inequalities for every $(i, j, t)$, and each index $n \in \mathbf{N}_{i j}$ is associated with an integer parameter $\mu_{i j n}$ (that may be different from the parameter $v_{i j n}$ used in (SII)). Parameter $\mu_{i j n}$ is the largest $\omega b_{i j n} i^{\prime}$ over index $i^{\prime}$ that appears in the second summation.

Similarly to (19), $\mu_{i j 1}$ is set to the smallest processing time of any $i^{\prime}$,

$$
\mu_{i j 1}=\min _{i \neq i} \tau_{i^{\prime} j}
$$

The value of $\mu_{i j, n+1}$ is chosen according to (23), so that no variables corresponding to changeover time points are excluded in (21). 


$$
\mu_{i j, n+1}=\min \left\{\left\{\mu_{i j n}+\min _{i^{\prime}: \mu_{i j n}<\sigma_{i^{\prime} i j}} \tau_{i^{\prime} j}, \max _{i^{\prime} \neq i} \sigma_{i^{\prime}}{ }_{i j}\right\}\right.
$$

Note that (23) is similar to (20), defining $v_{i j, n+1}$, except that (23) requires that $\mu_{i j, n+1}$ with the largest $\mathrm{n}$ is equal to the largest changeover time from any other task $i^{\prime}$ to task $i$, which leads to tighter constraints.

Before specifying $\omega a_{i j n} i^{\prime}$ and $\omega b_{i j n} i^{\prime}$ and thus completing constraints (21), we introduce two disjoint task subsets: $\mathbf{I A}_{i j n}=\left\{i^{\prime} \mid i^{\prime} \neq i, \mu_{i j n} \leq \sigma_{i^{\prime} i j}\right\}$ and $\mathbf{I B}_{i j n}=\left\{i^{\prime} \mid i^{\prime} \neq i, \mu_{i j n}>\sigma_{i^{\prime}}{ }^{\prime}\right\}$.

Parameters $\omega a_{i j n} i^{\prime}$ and $\omega b_{i j n} i^{\prime}$ depend on the subset task $i^{\prime}$ belongs to, and are defined as follows,

$$
\begin{aligned}
& \omega a_{i j n i^{\prime}}= \begin{cases}\tau_{i^{\prime} j}+\mu_{i j n}-1, & i^{\prime} \in \mathbf{I A}_{i j n} \\
\tau_{i^{\prime}{ }_{j}}+\sigma_{i^{\prime} i j}-1, & i^{\prime} \in \mathbf{I B}_{i j n}\end{cases} \\
& \omega b_{i j n i^{\prime}}=\left\{\begin{array}{cl}
\mu_{i j n}, & i^{\prime} \in \mathbf{I} \mathbf{A}_{i j n} \\
\max \left[\sigma_{i^{\prime} i j}, \mu_{i j n}-\min _{i^{\prime \prime} \in \mathbf{I} \mathbf{A}_{i j n}} \sigma_{i^{\prime \prime}} i^{\prime} j{ }^{\prime \prime}{ }_{i^{\prime \prime} \in \mathbf{I B}_{i j n} \backslash\left\{i^{\prime}\right\}}\left(\sigma_{i^{\prime \prime} i j}-\sigma_{i^{\prime \prime} i^{\prime} j}\right)\right\}, & i^{\prime} \in \mathbf{I} \mathbf{B}_{i j n}
\end{array}\right.
\end{aligned}
$$

The definitions in (24) are discussed next.

(1) If $i^{\prime} \in \mathbf{I} \mathbf{A}_{i j n}$, there are $\tau_{i^{\prime} j}$ variables $X_{i^{\prime}}{ }^{\prime} t^{\prime}$ in (21), which is the maximum number of variables to be included for a task; including more than $\tau_{i^{\prime}}{ }^{\prime}{ }^{\prime}$-indexed variables will make the constraint invalid, because it would cut off the solution in which task $i^{\prime}$ is carried out back-to-back.

(2) If $i^{\prime} \in \mathbf{I B}_{i j n}$, the smallest index $t^{\prime}$ for variables $X_{i^{\prime}}{ }^{\prime} t^{\prime}$ included in the constraint is the earliest changeover time point $\left(t^{\prime}=t-\tau_{i^{\prime}{ }_{j}}-\sigma_{i^{\prime}}{ }_{i j}+1\right)$ and the largest $t^{\prime}$ for included $X_{i^{\prime}}{ }^{\prime} t^{\prime}$ variables must be one period before the smallest of the following three (so that the constraint does not exclude any feasible sequences while remaining as tight as possible):

(2a) $t^{\prime}$ which would lead to the inclusion of exactly $\tau_{i^{\prime} j}+1$ variables for task $i^{\prime}$ (if $\omega b_{i j n} i^{\prime}=$ $\left.\sigma_{i^{\prime} i j}\right)$;

(2b) $t^{\prime}$ that would make (21) cut off the solution where $i^{\prime \prime} \in \mathbf{I} \mathbf{A}_{i j n}$ takes place at $t-\tau_{i^{\prime \prime} j}-$ $\mu_{i j n}+1$ and $i^{\prime}$ takes place $\tau_{i^{\prime \prime} j}+\sigma_{i^{\prime \prime} i^{\prime} j}$ periods later (if $\omega b_{i j n i} i^{\prime}=\mu_{i j n}-\sigma_{i^{\prime \prime} i^{\prime} j}$ );

(2c) $t^{\prime}$ that would make (21) cut off the solution where another $i^{\prime \prime} \in \mathbf{I B}_{i j n}$ takes place at

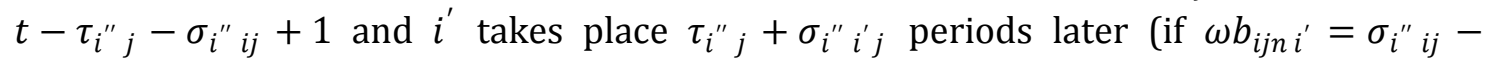
$\left.\sigma_{i}{ }^{\prime \prime} i^{\prime} j\right)$.

Table 3. Data for the 4-task example shown in Figure 4.

\begin{tabular}{llllll}
\hline & & \multicolumn{4}{l}{$\sigma_{i^{\prime} i}\left(i^{\prime}=\right.$ left, $\mathrm{i}=$ top $)$} \\
\cline { 3 - 6 } & $\tau_{\mathrm{i}}$ & $\mathrm{T} 1$ & $\mathrm{~T} 2$ & $\mathrm{~T} 3$ & $\mathrm{~T} 4$ \\
\hline $\mathrm{T} 1$ & 7 & 0 & 0 & 6 & 5 \\
$\mathrm{~T} 2$ & 4 & 1 & 0 & 3 & 1 \\
$\mathrm{~T} 3$ & 8 & 4 & 2 & 0 & 3 \\
$\mathrm{~T} 4$ & 6 & 2 & 2 & 2 & 0 \\
\hline
\end{tabular}

The proof of correctness of (SIIT) is presented in Appendix 2. Algorithm 1 summarizes the procedure for the calculation of $\omega a_{i j n i^{\prime}}$ and $\omega b_{i j n i^{\prime}}$ used in (SIIT). Table 3 gives the data for the example in Figure 4, with hollow points illustrating which variables are included in (SIIT). Figure 5 
presents a simple example illustrating how constraints (SIIT) cut off fractional solutions that are feasible for the LP-relaxation of other formulations.

Algorithm 1. Procedure to generate the parameters and sets used in (SIIT).

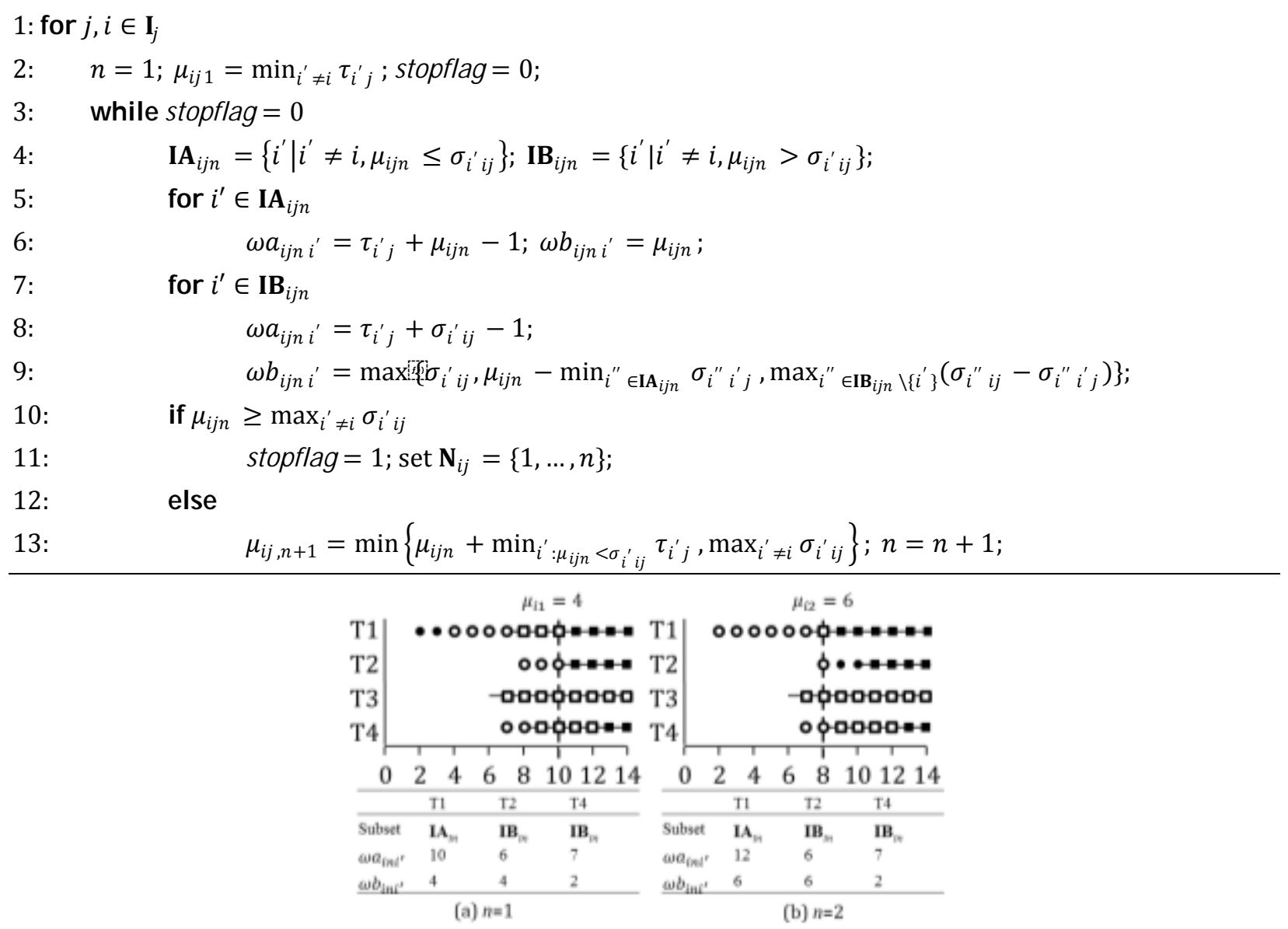

Figure 4. Parameters and constraints (SIIT) for $i=\mathrm{T} 3, t=14$, based on the data of Table 3 .

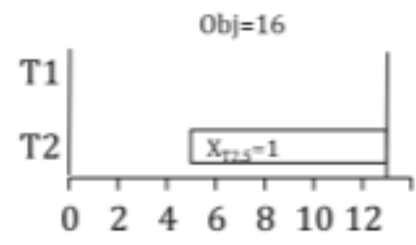

(a) Constraints (SIIT)

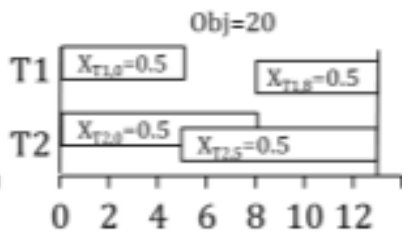

(b) Other constraints

Figure 5. Illustration of tightness of constraints (SIIT) for profit maximization.

Note. Two tasks, T1 $\left(\tau_{1}=5\right)$ and T2 $\left(\tau_{2}=8\right)$, are to be scheduled on a single unit; with changeover times $\sigma_{\mathrm{T} 1, \mathrm{~T} 2}=3$ and $\sigma_{\mathrm{T} 2, \mathrm{~T} 1}=2$; the horizon is 13 hours; and the profit from running $\mathrm{T} 1$ and $\mathrm{T} 2$ are 4 and 16 , respectively. The LP-relaxation with constraints (SIIT) yields the (optimal) integer solution, shown in (a). The solution of the LP-relaxation with all other changeover constraints, shown in (b), is cut off by constraints (21) with $i=\mathrm{T} 1, t=8, n=1\left(\omega a_{\mathrm{T} 1,1, \mathrm{~T} 2}=9, \omega b_{\mathrm{T} 1,1, \mathrm{~T} 2}=2\right)$.

Theorem 1 establishes that (SIIT) are facet-defining for the problem containing constraints (7) and (21) (proof in Appendix 3). 
Theorem 1: Let $H=\left\{X \in\{0,1\}^{T \cdot|\mathbf{I}| \cdot|\mathbf{I}|}\right.$ : subject to constraints (7) (21) $\}$. Then each inequality in (21) is facet-defining for the convex hull of $\mathrm{H}, \operatorname{conv}(\mathrm{H})$.

\subsection{Constraints (SIII)}

To write a constraint for a single time point, we sum constraints (SII) over $\mathrm{n}$ to obtain a big-M constraint with $\left|\mathbf{N}_{i j}\right|$ as the big-M parameter,

$$
\sum_{i \neq i t^{\prime}=t-\tau_{i^{\prime} j}-\sigma_{i^{\prime} i j}+1} \sum_{i^{\prime} j t^{\prime}}^{t-\tau_{i^{\prime} j}} \leq\left|\mathbf{N}_{i j}\right|\left(1-X_{i j t}\right) \quad \forall i, j, t
$$

Even if the binary variable $X_{i^{\prime}}{ }_{j t}{ }^{\prime}$ is included in multiple inequalities of (SII), we include it only once in (SIII) because we found that the tighter constraints (including binary variables multiple times) do not improve solution times (see Supplementary Material). Constraints (SIII) have the same form as (K). Figure 6a shows (SIII) for $i=\mathrm{T} 3, t=14$, with hollow points showing which binary variables are included in the constraint. Figure $6 \mathrm{~b}$ presents a Gantt chart where the constraint is binding, showing that, for this example with $i=\mathrm{T} 3$, the value of big-M is the smallest possible.

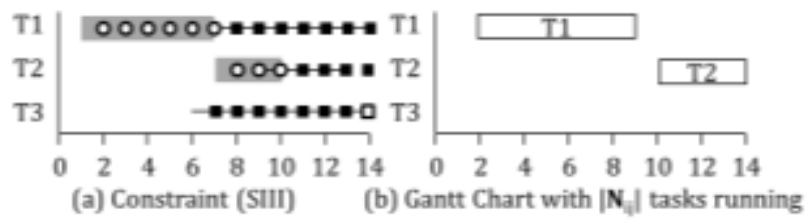

Figure 6. Illustration of constraints (SIII). (a) For $i=\mathrm{T} 3, t=14$, based on the data of Table 2. (b) A schedule in which (SIII) are binding.

\subsection{Constraints (PI), (PII), and (PIII)}

To formulate constraints (PI), we start by writing constraints for a pair of tasks $\left(i, i^{\prime}\right)$ based on (SI)

$$
\begin{gathered}
X_{i j t}+\sum_{t^{\prime \prime}=\max \left\{t^{\prime}, t-\sigma_{i^{\prime} i j}\right\}-\tau_{i^{\prime} j}+1}^{\min \left\{t^{\prime}, t-\tau_{i^{\prime} j}\right\}} X_{i^{\prime} j t^{\prime \prime}} \leq 1 \\
\forall i, i^{\prime} \neq i, j, t, t-\max \left\{\min _{i^{\prime} \neq i} \tau_{i^{\prime} j}, \max _{i \neq i} \sigma_{i^{\prime}}{ }_{i j}\right\} \leq t^{\prime} \leq t-\min _{i \neq i} \tau_{i^{\prime} j}
\end{gathered}
$$

Since the task $i^{\prime}$ is specified, we have $t^{\prime}=t-\tau_{i^{\prime} j}$, as

$$
t-\max \left\{\min _{i \neq i} \tau_{i^{\prime} j} \max _{i \neq i} \sigma_{i^{\prime}}{ }_{i j}\right\} \leq t^{\prime} \leq t-\min _{i \neq i} \tau_{i^{\prime} j} \Leftrightarrow t^{\prime}=t-\tau_{i^{\prime} j}
$$

Since there is only one possible time point, $t^{\prime}$, for which we can write constraints (PI), the three pairwise constraints, (PI), (PII), and (PIII), will all be the same. For $t^{\prime}=t-\tau_{i^{\prime}{ }_{j}}$, we have

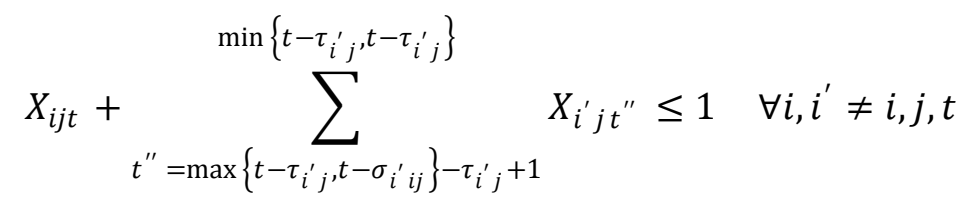

Because $\tau_{i^{\prime}}>\sigma_{i^{\prime} i j}$ (Assumption 1), this reduces to 


$$
X_{i j t}+\sum_{t^{\prime \prime}=t-\tau_{i^{\prime} j}-\sigma_{i^{\prime} i j}+1}^{t-\tau_{i^{\prime} j}} X_{i^{\prime} j t^{\prime \prime}} \leq 1 \quad \forall i, i^{\prime} \neq i, j, t
$$

which is (PI), (PII), and (PIII) and will be referred to as constraints (P). The hollow points in Figure 7 show which binary variables are included in the constraints for $i=\mathrm{T} 3, t=14$, and $i^{\prime}=\mathrm{T} 1$ or $\mathrm{T} 2$.

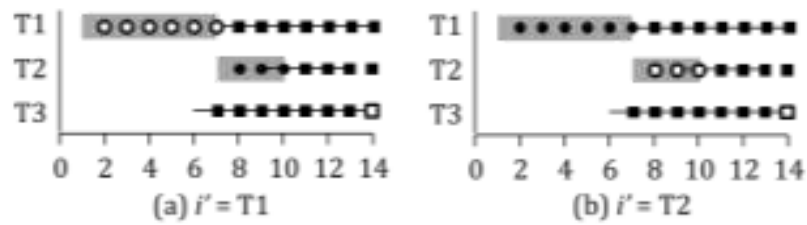

Figure 7. Constraints (P) for $i=\mathrm{T} 3, t=14$ and $i^{\prime}=\mathrm{T} 1$ and $\mathrm{T} 2$, based on the data of Table 2 .

\section{Remarks}

\subsection{Tightening}

Some of the variables included in the assignment constraints (7) can be included in the LHS of the changeover constraints without increasing the right hand side (RHS), thereby tightening the constraints. These constraints are presented for completeness, but not studied further because they were found to be less effective (based on our computational study, they are up to 5 times slower). All variables indexed by $i$ and included in the assignment constraint at time $t$ can be included in (SI), (SII), (SIII) and (P). For (SI), we raise the upper bound on the summation over $t^{\prime \prime}$ to $t^{\prime}$ and change the bounds on $t^{\prime}$ to include more pairs of time points.

$$
\sum_{t^{\prime \prime}=t-\tau_{i j}+1}^{t} X_{i j t^{\prime \prime}}+\sum_{i^{\prime} \neq i} \sum_{t^{\prime \prime}=\max }^{t^{\prime}} \sum_{\left.t^{\prime}, t-\sigma_{i^{\prime} i j}\right\}-\tau_{i^{\prime} j}+1} X_{i^{\prime} j t^{\prime \prime}} \leq 1 \forall i, j, t, t-\max _{i^{\prime} \neq i} \sigma_{i^{\prime} i j} \leq t^{\prime} \leq t-1
$$

For (SII), we use the same values for $v_{\mathrm{ijn}}$, but we raise the upper bound on the summation over $t^{\prime}$ :

$$
\sum_{t^{\prime}=t-\tau_{i j}+1}^{t} X_{i j t^{\prime}}+\sum_{i^{\prime} \neq i} \sum_{t^{\prime}=\max \left\{t-v_{i j n}, t-\sigma_{i^{\prime} i j}\right\}-\tau_{i^{\prime} j}+1}^{t-v_{i j n}} X_{i^{\prime} j t^{\prime}} \leq 1 \quad \forall i, j, t, n \in \mathbf{N}_{i j}
$$

For (SIII), we only include the additional variables for task $i$.

$$
\sum_{i^{\prime} \neq i} \sum_{t^{\prime}=t-\tau_{i^{\prime} j}-\sigma_{i^{\prime} i j}+1}^{t-\tau_{i^{\prime} j}} X_{i^{\prime} j t^{\prime}} \leq\left|\mathbf{N}_{i j}\right| \cdot\left(1-\sum_{t^{\prime}=t-\tau_{i j}+1}^{t} X_{i j t^{\prime}}\right) \quad \forall i, j, t
$$

For (P), we raise the upper bound on $t^{\prime \prime}$ in the summation to include times up to $t-\sigma_{i^{\prime} i j}$.

$$
\sum_{t^{\prime}=t-\tau_{i j}+1}^{t} X_{i j t^{\prime}}+\sum_{t^{\prime \prime}=t-\tau_{i^{\prime} j}-\sigma_{i^{\prime} i j}+1}^{t-\sigma_{i^{\prime} i j}} X_{i^{\prime} j t^{\prime \prime}} \leq 1 \quad \forall i, i^{\prime} \neq i, j, t
$$


Finally the same idea can be applied to (K). However, after including the additional variables, parameter M needs to be modified,

$$
\sum_{i^{\prime} \neq i} \sum_{t^{\prime}=t}^{t+\tau_{i j}+\sigma_{i i^{\prime} j}-1} X_{i^{\prime} j t^{\prime}} \leq\left\{\sum_{i^{\prime} \neq i}\left\lceil\frac{\tau_{i j}+\sigma_{i i^{\prime} j}}{\tau_{i^{\prime} j}}\right]\right\} \cdot\left(1-X_{i j t}\right) \quad \forall i, j, t
$$

\subsection{Assumptions}

Some of the assumptions of changeover times can be relaxed for some of the formulations.

Assumption 1. When Assumption 1 does not hold, constraints (SI), (SII), (SIII), (K), (SH) and (W) are valid without any changes; constraints (P) are not valid, but can be rewritten to ensure validity. In this case, (PI)-(PIII) will be different. (PI) become

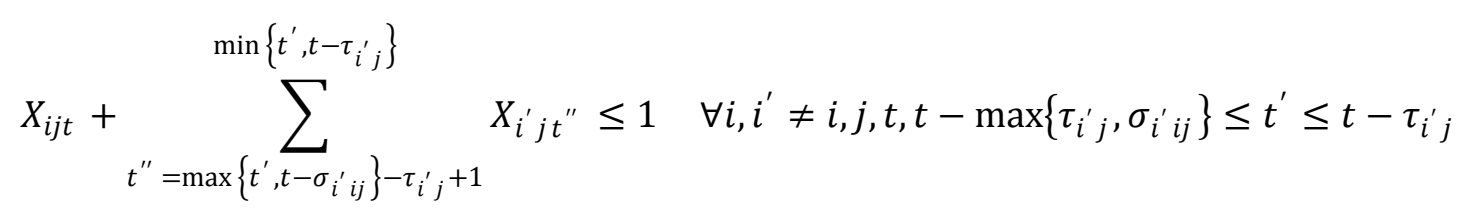

(PII) include (PI) for $t^{\prime}=t-n \tau_{i^{\prime} j}$ where $n=1,2, \ldots,\left\lceil\sigma_{i^{\prime} i j} / \tau_{i^{\prime} j}\right\rceil$

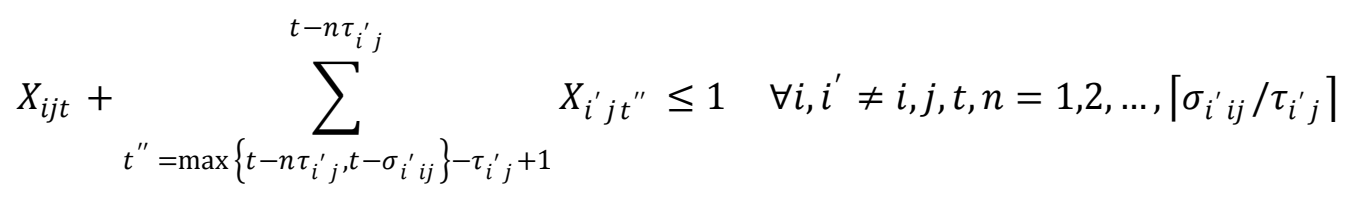

(PIII) have the same form as (P), but with a different coefficient on $X_{i j t}$ and a different constant.

$$
\sum_{t^{\prime \prime}=t-\tau_{i^{\prime} j}-\sigma_{i^{\prime} i j}+1}^{t-\tau_{i^{\prime} j}} X_{i^{\prime} j t^{\prime \prime}} \leq\left\lceil\sigma_{i^{\prime}{ }^{\prime}{ }_{j}} / \tau_{i^{\prime}{ }^{\prime}}\right\rceil \cdot\left(1-X_{i j t}\right) \quad \forall i, i^{\prime} \neq i, j, t
$$

Assumption 2. If Assumption 2 does not hold, all presented constraints, including (K), (SH) and (W), will allow a short task to run during the changeover between two other tasks (Figure 8). Zentner et al. (1994) presented a formulation that is valid when this assumption does not hold.

Assumption 3. For all changeover constraints except (W), which are valid only when each task is restricted to run once, Assumption 3 can be removed by including task $i$ in all summations, minimizations, and maximizations over all $i^{\prime}$ instead of $i^{\prime} \neq i$, and by writing the pairwise constraints for task $i$ and all $i^{\prime}$. For example, (SI) become

$$
\begin{aligned}
& X_{i j t}+\sum_{i^{\prime}} \sum_{t^{\prime \prime}=\max \left\{t^{\prime}, t-\sigma_{i^{\prime} i j}\right\}-\tau_{i^{\prime} j}+1}^{\min \left\{t^{\prime}, t-\tau_{i^{\prime} j}\right\}} X_{i^{\prime} j t^{\prime \prime}} \leq 1 \\
& \left.\forall i, j, t, t-\max \left\{\min _{i} \tau_{i^{\prime} j}, \max _{i} \sigma_{i^{\prime}}{ }^{\prime}\right\}\right\} \leq t^{\prime} \leq t-\min _{i} \tau_{i^{\prime} j}
\end{aligned}
$$




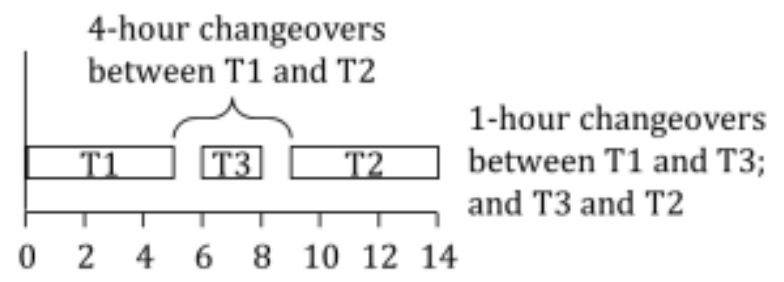

Figure 8. When the triangle inequality is not satisfied, task T3 with processing time of 2 hours can run during the changeover between $\mathrm{T} 1$ and $\mathrm{T} 2$.

\subsection{Valid Inequalities}

Velez and Maravelias presented valid inequalities that are effective at reducing the solution time for makespan minimization problems (Velez and Maravelias, 2013). We modify this inequality to consider the effect of changeovers:

$$
M S \geq \sum_{i, t} \tau_{i j} X_{i j t}+\sum_{i^{\prime}, t}\left(\min _{i \neq i} \sigma_{i^{\prime}}{ }_{i j}\right) X_{i^{\prime} j t}-\max _{i}\left(\min _{i \neq i} \sigma_{i^{\prime} i j}\right) \quad \forall j
$$

The first term in the RHS represents the total processing time on unit $j$, and the second term is the minimum changeover time after any task $i^{\prime}$. At the end of the horizon, we do not need to include a changeover, so the final term subtracts the maximum time that could have been added to the second term. When a task can be executed multiple times, we only use the first term on the RHS of constraints (36), because task $i$ is allowed to immediately follow itself without any changeovers.

Constraints (36) improve the lower bound on the makespan and allow integer solutions to be found faster (see §6). The makespan for the LP-relaxation is minimized when all $X_{i j t}$ are small and nonzero (see inequalities (3)), leading to many fractional binary variables. In (36), the makespan depends on the sum, over $i$ and $\mathrm{t}$, of $X_{i j t}$ for each unit j. For example, in the single-unit problem the lower bound on the makespan using (36) is the same regardless of whether $X_{i t}$ is one for a single time point or fractional for many, and thus the sum of $X_{i t}$ over t remains 1 for each $i$, which makes (36) tighter than (3) in this case.

\section{Relative Tightness of Formulations}

We evaluate the relative tightness of the new formulations and the three from the literature. To show that one formulation is at least as tight as another, we prove that any point that is feasible for the LP-relaxation of the tighter formulation is also feasible for the other formulation. To show a formulation is tighter, we find a point for a specific instance that is feasible for the less tight formulation, but not for the tighter one. These points are given in Supplementary Material. In some cases, neither formulation is tighter, so we find a point that satisfies either one but not the other.

The binary variables included in $(\mathrm{K})$ are for time points after $\mathrm{t}$, while all other changeover constraints include binary variables before t. For consistency, we will use the backwards version of $(\mathrm{K})$, henceforth referred to as (KB), where we use $\mathrm{M}$ that is the forward analog of constraints (13): 


$$
\sum_{i \neq i} \sum_{t^{\prime}=t-\tau_{i^{\prime} j}-\sigma_{i^{\prime} i j}+1}^{t-\tau_{i^{\prime} j}} X_{i^{\prime} j} t^{\prime} \leq\left(\sum_{i^{\prime} \neq i} \sigma_{i^{\prime}}{ }^{i j}\right) \cdot\left(1-X_{i j t}\right) \quad \forall i, j, t
$$

Since (K) use the M given by expression (14), all proofs will be based on this value; but we note that using a smaller value for $\mathrm{M}$ may change the relative tightness of two constraints. Also, similarly to $(\mathrm{K})$, all constraints could be written looking forward in time. In general neither type of constraints is tighter than the other.

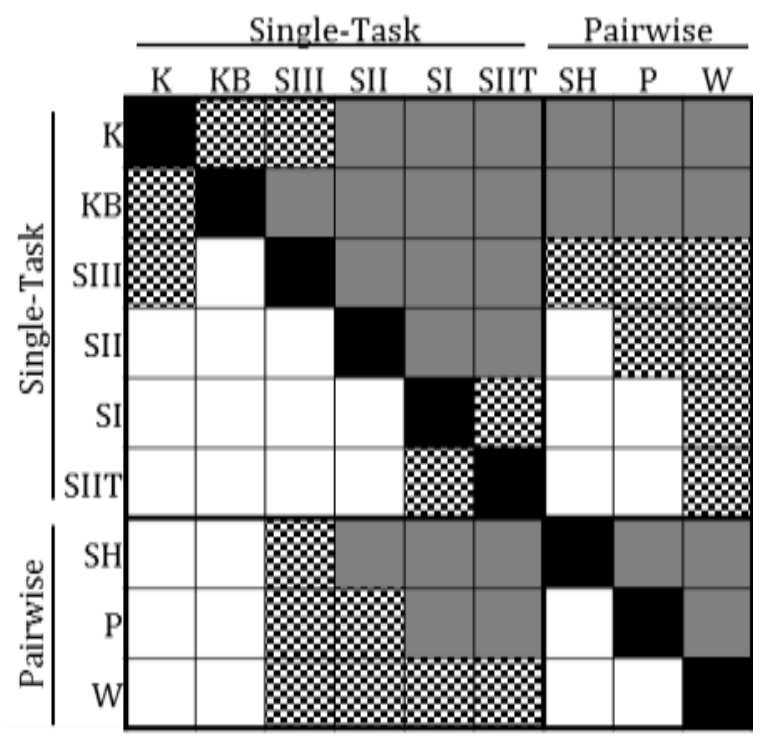

Figure 9. Relative tightness of all formulations.

Figure 9 summarizes the relative tightness of all formulations. White indicates that the formulation on the left is tighter than the formulation on the top; gray indicates the opposite. A dotted block indicates that neither formulation is tighter. Constraints (W) are the tightest among the pairwise changeover constraints and (SI) and (SIIT) are the tightest among the single-task changeover constraints. In most cases neither the pairwise nor the single-task constraints are tighter. Singletask constraints have the advantage of including binary variables for many different tasks in a single constraint. Pairwise constraints have the advantage that they can include binary variables for more time points for a single task in a single constraint.

Proposition 1: $(\mathrm{SH})$ are tighter than $(\mathrm{K}) /(\mathrm{KB})$.

Proof: Consider any point satisfying (SH). For fixed $i^{\prime}, j$ and $t^{\prime}$, we add (SH) over all $i \neq i^{\prime}$ and all $t \in\left\{t^{\prime}+\tau_{i^{\prime}{ }^{\prime}}, \ldots, t^{\prime}+\tau_{i^{\prime} j}+\sigma_{i^{\prime} i j}-1\right\}$ to get

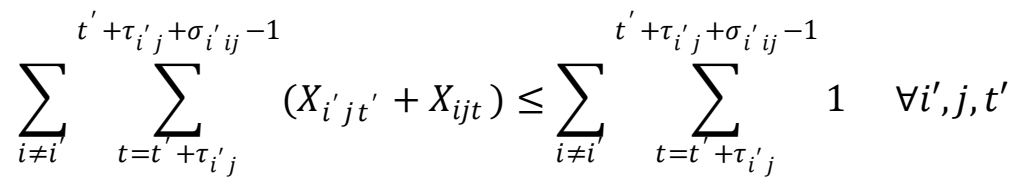




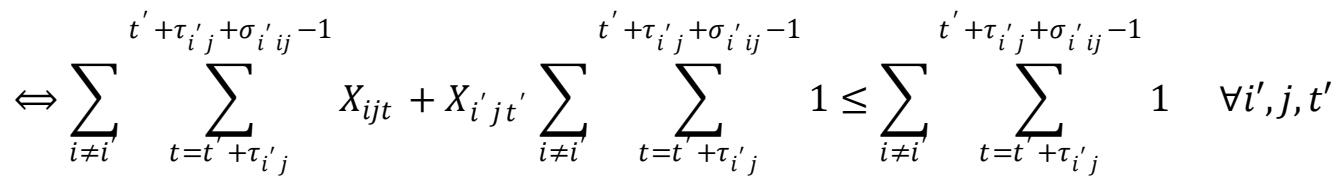

The sums in the second term on the LHS and on the RHS are equal to $M_{i^{\prime} j}$ in expression (14).

$$
\begin{aligned}
& \Leftrightarrow \sum_{i \neq i} \sum_{t=t^{\prime}+\tau_{i^{\prime} j}}^{t^{\prime}+\tau_{i^{\prime}}+\sigma_{i^{\prime}{ }^{\prime} j}-1} X_{i j t} \leq M_{i^{\prime} j}-M_{i^{\prime} j} X_{i^{\prime} j t^{\prime}} \quad \forall i^{\prime}, j, t^{\prime} \\
& \Leftrightarrow \sum_{i \neq i} \sum_{t=t^{\prime}+\tau_{i^{\prime} j}}^{t^{\prime}+\tau_{i^{\prime} j^{\prime}+\sigma_{i^{\prime} i j}-1}} X_{i j t} \leq M_{i^{\prime} j}\left(1-X_{i^{\prime} j t^{\prime}}\right) \quad \forall i^{\prime}, j, t^{\prime}
\end{aligned}
$$

Switching $i$ with $i^{\prime}$ and t with $t^{\prime}$ gives (K). Therefore, any point satisfying (SH) also satisfies (K).

A similar proof shows that (SH) are also tighter than (KB). If we use any smaller value for $M_{i j}$ in $(\mathrm{K})$, then neither one is tighter.

Proposition 2: (SIII) are tighter than (KB).

Proof: Consider any point satisfying (SIII). The value of $\left|\mathbf{N}_{i j}\right|$ in (SIII) is at least as small as the value of $\mathrm{M}$ in (KB), and for given $i, \mathrm{j}$ and $\mathrm{t}$, the constraint of (SIII) contains the same binary variables as (KB).

$$
\sum_{i \neq i t^{\prime}=t-\tau_{i^{\prime} j}-\sigma_{i^{\prime} i j}+1}^{t-\tau_{i^{\prime} j}} X_{i^{\prime} j t^{\prime}} \leq\left|\mathbf{N}_{i j}\right|\left(1-X_{i j t}\right) \leq M_{i j}\left(1-X_{i j t}\right) \quad \forall i, j, t
$$

Therefore, any point satisfying (SIII) also satisfies (KB).

Proposition 3: (SII) are tighter than (SIII).

Proof: Any point satisfying (SII) also satisfies (SIII), since (SIII) are formulated by combining constraints from (SII) and then lowering some of the coefficients in the aggregate inequality (for $X_{i}{ }^{\prime} j t^{\prime}$ included in (SII) for multiple values of $\mathrm{n}$ ).

Proposition 4: (SII) are tighter than (SH).

Proof: Consider any point satisfying (SII). The constraint of (SII) for $\mathrm{n}$ contains both variables in (SH) and therefore dominates (SH) for $t^{\prime} \in\left\{t-\min \left\{\sigma_{i^{\prime} i j}, v_{i j n}\right\}-\tau_{i^{\prime} j}+1, \ldots, t-\max \left\{\tau_{i^{\prime} j}, v_{i j n}\right\}\right\}$

$$
\begin{gathered}
X_{i j t}+X_{i^{\prime} j t^{\prime \prime}} \leq X_{i j t}+\sum_{i \neq i} \sum_{t^{\prime}=\max \left\{t-v_{i j n}, t-\sigma_{i^{\prime}{ }^{\prime} j}\right\}-\tau_{i^{\prime} j^{\prime}}+1}^{\min \left\{t-v_{i j n}, t-\tau_{i^{\prime} j}\right\}} X_{i^{\prime} j t^{\prime}} \leq 1 \\
\forall i, j, t, n \in \mathbf{N}_{i j}, t^{\prime \prime} \in\left\{t-\min \left\{\sigma_{i^{\prime}}{ }_{i j}, v_{i j n}\right\}-\tau_{i^{\prime} j}+1, \ldots, t-\max \left\{\tau_{i^{\prime}{ }^{\prime}}, v_{i j n}\right\}\right\}
\end{gathered}
$$

All variables $X_{i^{\prime}}{ }^{\prime} t^{\prime}$ with $t^{\prime}$ that are included in (SH) are included in the given interval for some $\mathrm{n}$. 
$\bigcup_{n \in \mathbf{N}_{i j}}\left\{t-\min \left\{\sigma_{i^{\prime} i j}, v_{i j n}\right\}-\tau_{i^{\prime} j}+1, \ldots, t-\max \left\{\tau_{i^{\prime}{ }^{\prime}}, v_{i j n}\right\}\right\}=\left\{t-\sigma_{i^{\prime} i j}-\tau_{i^{\prime} j}+1, \ldots, t-\tau_{i^{\prime} j}\right\}$

Therefore, any point satisfying (SII) also satisfies (SH).

Proposition 5: (SI) are tighter than (SII).

Proof: Consider any point satisfying (SI). The constraints of (SI) for $t^{\prime}=t-v_{i j n}$ for $n=$ $1,2, \ldots,\left|\mathbf{N}_{i j}\right|-1$ are also included in (SII). Additionally, the constraint of (SI) for the smallest $t^{\prime}$

$$
X_{i j t}+\sum_{i \neq i t^{\prime \prime}=t-\sigma_{i^{\prime} i j}-\tau_{i^{\prime} j}+1}^{\min \left\{t^{\prime}, t-\tau_{i^{\prime} j}\right\}} X_{i^{\prime} j t^{\prime \prime}} \leq 1 \quad \forall i, j, t, t^{\prime}=t-\max \left\{\min _{i \neq i} \tau_{i^{\prime} j}, \max _{i^{\prime} \neq i} \sigma_{i^{\prime}}{ }_{i j}\right\}
$$

dominates the constraint of (SII) for $n=\left|\mathbf{N}_{i j}\right|$

$$
X_{i j t}+\sum_{i \neq i} \sum_{t^{\prime}=t-\sigma_{i^{\prime}{ }^{\prime} j^{\prime}}-\tau_{i^{\prime} j}+1}^{\min \left\{t-v_{i j n}, t-\tau_{i^{\prime} j}\right\}} X_{i^{\prime} j t^{\prime}} \leq 1 \quad \forall i, j, t, n=\left|\mathbf{N}_{i j}\right|
$$

Because

$$
v_{i j,\left|\mathbf{N}_{i j}\right|} \geq \min _{i \neq i} \tau_{i^{\prime} j} \text { and } v_{i j,\left|\mathbf{N}_{i j}\right|} \geq \max _{i^{\prime} \neq i} \sigma_{i^{\prime} i j} \quad \forall j
$$

which means

$$
t-v_{i j,\left|\mathbf{N}_{i j}\right|} \leq t^{\prime} \text { and } \min \left\{t-\tau_{i^{\prime}{ }^{\prime}}, t-v_{i j,\left|\mathbf{N}_{i j}\right|}\right\} \leq \min \left\{t t-\tau_{i^{\prime}{ }^{\prime}}, t^{\prime}\right\}
$$

and

$$
\begin{aligned}
& X_{i j t}+\sum_{i \neq i t^{\prime \prime}=t-\tau_{i^{\prime} j}-\sigma_{i^{\prime} i j}+1}^{\min \left\{t-v_{i j n}, t-\tau_{i^{\prime} j}\right\}} X_{i^{\prime} j t^{\prime \prime}} \leq X_{i j t}+\sum_{i^{\prime} \neq i t^{\prime \prime}=t-\tau_{i^{\prime} j}-\sigma_{i^{\prime}{ }^{\prime} j}+1} \sum_{i^{\prime} j t^{\prime \prime}}^{\min \left\{t^{\prime}, t-\tau_{i^{\prime} j}\right\}} \sum_{1} \\
& \forall i, j, t, n=\left|\mathbf{N}_{i j}\right|, t^{\prime}=t-\max \left\{\min _{i \neq i} \tau_{i^{\prime} j}, \max _{i \neq i} \sigma_{i^{\prime}}{ }^{\prime} i j\right\}
\end{aligned}
$$

Therefore, any point satisfying (SI) also satisfies (SII).

Proposition 6: (P) are tighter than (SH).

Proof: Consider any point satisfying (P). One variable in the sum is less than the entire sum, if all variables in the sum are non-negative.

$$
X_{i^{\prime} j t^{\prime}} \leq \sum_{t^{\prime \prime}=t-\tau_{i^{\prime} j}-\sigma_{i^{\prime} i j}+1}^{t-\tau_{i^{\prime} j}} X_{i^{\prime} j t^{\prime \prime}} \leq 1-X_{i j t} \quad \forall i, i^{\prime} \neq i, j, t, t-\tau_{i^{\prime} j}-\sigma_{i^{\prime} i j}<t^{\prime} \leq t-\tau_{i^{\prime} j}
$$

Therefore, any point satisfying (P) also satisfies (SH).

Proposition 7: (W) are tighter than (P).

Proof: Consider any point satisfying (W). 


$$
\begin{gathered}
\sum_{t^{\prime}=t-\tau_{i j}-\sigma_{i i^{\prime} j}+1}^{t} X_{i j t^{\prime}}+\sum_{t^{\prime}=t-\tau_{i^{\prime} j}-\sigma_{i^{\prime} i j}+1}^{t} X_{i^{\prime} j t^{\prime}} \leq 1 \quad \forall i, i^{\prime} \neq i, j, t \\
\Leftrightarrow X_{i j t}+\sum_{t^{\prime}=t-\tau_{i j}-\sigma_{i i^{\prime} j}+1}^{t-1} X_{i j t^{\prime}}+\sum_{t^{\prime}=t-\tau_{i^{\prime} j}-\sigma_{i^{\prime} i j}+1}^{t-\tau_{i^{\prime} j}} X_{i^{\prime} j t^{\prime}}+\sum_{t^{\prime}=t-\tau_{i^{\prime} j}+1}^{t} X_{i^{\prime} j t^{\prime}} \leq 1 \quad \forall i, i^{\prime} \neq i, j, t \\
\Rightarrow X_{i j t}+\sum_{t^{\prime}=t-\tau_{i^{\prime}{ }^{\prime} j}-\sigma_{i^{\prime}{ }^{\prime} i j}+1}^{t} X_{i^{\prime} j t^{\prime}} \leq 1 \quad \forall i, i^{\prime} \neq i, j, t
\end{gathered}
$$

Therefore, any point satisfying (W) also satisfies (P).

Proposition 8: (SI) are tighter than (P).

Proof: Consider any point satisfying (SI).

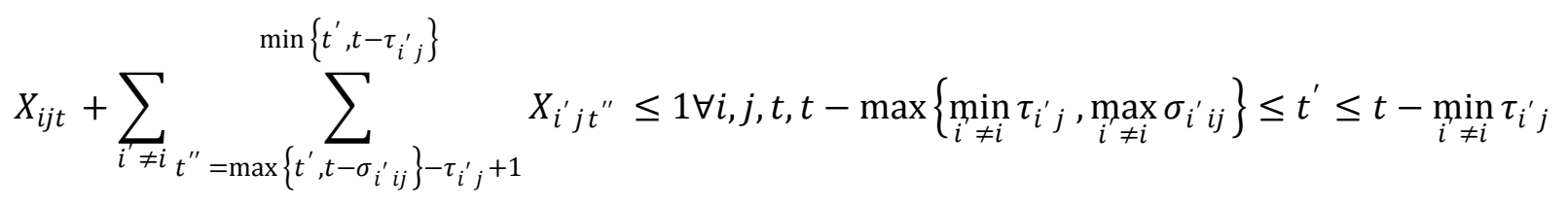

Removing the sum over $i^{\prime}$ gives a smaller value for the LHS.

$$
\begin{gathered}
X_{i j t}+\sum_{t^{\prime \prime}=\max \left\{t^{\prime}, t-\sigma_{i^{\prime}{ }^{\prime} i j}\right\}-\tau_{i^{\prime} j}+1}^{\min \left\{t^{\prime}, t-\tau_{i^{\prime} j}\right\}} X_{i^{\prime} j t^{\prime \prime}} \leq 1 \\
\forall i, i^{\prime} \neq i, j, t, t-\max \left\{\min _{i \neq i} \tau_{i^{\prime} j}, \max _{i^{\prime} \neq i} \sigma_{i^{\prime} i j}\right\} \leq t^{\prime} \leq t-\min _{i \neq i} \tau_{i^{\prime} j}
\end{gathered}
$$

The constraint for $t^{\prime}=t-\max \left\{\min _{i^{\prime \prime} \neq i} \tau_{i}{ }^{\prime \prime}{ }_{j}, \sigma_{i^{\prime}}{ }_{i j}\right\}$ is identical to the constraint of (P).

$$
\Leftrightarrow X_{i j t}+\sum_{t^{\prime \prime}=t-\tau_{i^{\prime} j}-\sigma_{i^{\prime} i j}+1}^{t-\tau_{i^{\prime} j}} X_{i^{\prime} j t^{\prime \prime}} \leq 1 \quad \forall i, i^{\prime} \neq i, j, t
$$

Therefore, any point satisfying (SI) also satisfies (P).

Proposition 9: (SIIT) are tighter than (SII).

Proof: Consider any point satisfying (SIIT). From the definitions of $\omega a_{i j n} i^{\prime}$ and $\omega b_{i j n} i^{\prime}$, we have that $t-\omega a_{i j n i^{\prime}}=\max \left\{t-\mu_{i j n}, t-\sigma_{i^{\prime} i j}\right\}-\tau_{i^{\prime} j}+1$, and $t-\omega b_{i j n i^{\prime}} \geq \min \left\{t-\mu_{i j n}, t-\tau_{i^{\prime} j}\right\}$.

When $n=1,2, \ldots,\left|\mathbf{N}_{i j}\right|-1, \mu_{i j n}=v_{i j n}$. Therefore, $t-\omega a_{i j n i^{\prime}}=\max \left\{t-v_{i j n}, t-\sigma_{i^{\prime} i j}\right\}-\tau_{i^{\prime} j}+1$ and $t-\omega b_{i j n i^{\prime}} \geq \min \left\{t-v_{i j n}, t-\tau_{i^{\prime} j}\right\}$.

When $n=\left|\mathbf{N}_{i j}\right|$, we have $\mu_{i j n} \leq v_{i j n}$ and $\sigma_{i^{\prime} i j} \leq \mu_{i j n}$. Therefore, $t-\omega a_{i j n i} i^{\prime}=t-\sigma_{i^{\prime} i j}-\tau_{i^{\prime} j}+1=$ $\max \left\{t-v_{i j n}, t-\sigma_{i^{\prime} i j}\right\}-\tau_{i^{\prime} j}+1$, and $t-\omega b_{i j n} i^{\prime} \geq \min \left\{t-\mu_{i j n}, t-\tau_{i^{\prime} j}\right\} \geq \min \left\{t-v_{i j n}, t-\tau_{i^{\prime} j}\right\}$. 
Thus, all binary variables included in (SII) on the LHS are also included in (SIIT), which leads to the conclusion that any point satisfying (SIIT) also satisfies (SII).

\section{Computational Study}

We tested several instances of the three problems we introduced in $§ 2$ : the single unit process, the parallel unit process, and the parallel unit process with unequal capacities. Different objective functions were also studied: tardiness minimization and cost minimization are relatively easier problems, so we considered instances with 5, 10, 15, 20, 25 tasks on 1, 3, 5 units. Makespan minimization is harder, so instances with $7,8,9,10,11$ tasks on $1,3,5$ units were studied. We used a step length of $\delta=1$ hour for all instances. Processing times were randomly selected from 3-9 hours (uniform distribution) and rounded up so that they are multiples of the step size, $\delta$, and costs for each task were randomly selected from \$1-10 (uniform distribution). For the tardiness minimization instances, due times were randomly selected between zero and the horizon length. For the cost minimization problem with deadlines (described in \$2.2), deadlines were generated based on the solution of tardiness minimization: if a task in the best solution is finished by the due time, then the due time of this task was used as its deadline; otherwise, we randomly selected the deadline between the finish time of the task and the horizon length. This adjustment was necessary to ensure that the cost minimization instances, with strict deadlines, were feasible. The length of the horizon is given in each subsection; unit capacities and customer demands, for processes with parallel units of unequal capacities are given in $§ 6.3$.

Changeover times were selected randomly so that they were less than some factor, $\varepsilon$, times the

minimum processing time of the two tasks, $\sigma_{i^{\prime}}{ }_{i j} \in\left[0, \varepsilon \cdot \min \left\{\tau_{i j}, \tau_{i^{\prime} j}\right\}\right]$. These changeover times may violate the triangle inequality from Assumption 2. If $\sigma_{i i}{ }_{j}>\sigma_{i i^{\prime}}+\tau_{i^{\prime} j}+\sigma_{i^{\prime}{ }^{\prime \prime} j}$, then we chose a new value $\sigma_{i i}{ }^{\prime \prime}{ }_{j} \in\left[0, \sigma_{i i}{ }^{\prime}{ }_{j}+\tau_{i^{\prime} j}+\sigma_{i^{\prime} i^{\prime \prime} j}\right]$. We updated the changeover times until all times satisfy the triangular inequality and rounded the changeover times so that they were multiples of the step size, $\delta$. We considered $\varepsilon=0.25,0.5,0.75$ and 1 . For a given number of tasks and units, and a given factor, 5 instances were generated. Thus, for makespan and tardiness minimization, we tested 500 instances, and for cost minimization 400 instances.

In this section, we refer to a specific model as $\mathrm{M}_{\mathrm{X}}^{\mathrm{Y}}$, where $\mathrm{X}$ denotes the changeover constraints, i.e., $\mathrm{X} \in\{\mathrm{K}, \mathrm{SH}, \mathrm{W}, \mathrm{SI}, \mathrm{SII}, \mathrm{SIII}, \mathrm{SIIT}, \mathrm{P}\}$; and Y denotes the objective function, which can be MS (makespan minimization), TRD (tardiness minimization), and CST (cost minimization). We also use MS-VI to denote makespan minimization with valid inequalities (36). For example, $\mathrm{M}_{\mathrm{SI}}^{\mathrm{MS}}$ is the model that includes constraints (SI) for makespan minimization. We use $\mathrm{M}_{\mathrm{X}}$ to denote all models with constraints $\mathrm{X}$, regardless of the objective function; and $\mathrm{M}^{\mathrm{Y}}$ to denote all models with objective function $Y$, regardless of changeover constraints.

All the instances were solved using CPLEX 12.6.3 via GAMS 24.7.1 on a cluster with 21 Intel Xeon (E5520) processors at $2.27 \mathrm{GHz}$ and $16 \mathrm{~GB}$ of RAM running on CentOS Linux 7, with a 1800-second resource limit. To better assess the effectiveness of the formulations we turned off the aggregator, 
presolver and presolver for initial relaxation by setting CPLEX options aggind, preind, and relaxpreind to zero. This set of CPLEX options is referred to as C1. Using CPLEX default settings, referred to as $\mathrm{C} 2$, requires less time, but leads to similar conclusions, which are briefly discussed in $\S 6.4$. Detailed results for C2 are given in Supplementary Material.

Finally, for constraints (K), we used a tight big-M, calculated using expression (38), though the performance is similar to the performance using $\mathrm{M}$ defined in expression (14).

$$
M_{i j}=\sum_{i \neq i}\left\lceil\frac{\sigma_{i i^{\prime} j}}{\tau_{i^{\prime} j}}\right\rceil
$$

\subsection{Single Unit}

The time horizon, $\eta$, is selected to ensure that there is enough time for all tasks to be completed. The calculation is based on the number of tasks and the longest processing time.

$$
\eta=\left(\max _{i} \tau_{i}\right) \cdot(2|\mathbf{I}|-1)
$$

The second term in the product is the number of tasks plus changeovers that take place. We multiplied this term by the maximum processing time.

The performance charts in Figure 10 show the fraction of instances that are solved by each model within the given amount of time (normalized to the fastest model for each instance). Figure 10a and 10c show the results for makespan minimization and tardiness minimization, respectively, using different changeover constraints (SI), (SII), (SIII), (P), (SIIT), (K), (SH), (W). We observe that although $\mathrm{M}_{\mathrm{W}}$ and $\mathrm{M}_{\mathrm{SIIT}}$ are the tightest models, they are also the slowest. Also, $\mathrm{M}_{\mathrm{SIII}}$ is one of the fastest models although it is among the weakest. Models $\mathrm{M}_{\mathrm{K}}, \mathrm{M}_{\mathrm{SI}}, \mathrm{M}_{\mathrm{SII}}, \mathrm{M}_{\mathrm{SIII}}$, whose changeover constraints are all written in terms of a single task, are much faster than $M_{P}, M_{S H}, M_{W}$, whose changeover constraints are written in terms of pairs of tasks. Figure $10 \mathrm{~b}$ shows the results for makespan minimization with the four fastest changeover constraints, (SIII), (SII), (SI) and (K), and with valid inequalities (36) included. Adding (36) does not lead to computational enhancements for single-unit problems. For makespan minimization, $\mathrm{M}_{\mathrm{SII}}^{\mathrm{MS}}$ and $\mathrm{M}_{\mathrm{SIII}}^{\mathrm{MS}}$ are the fastest models; while for tardiness minimization, $\mathrm{M}_{\mathrm{K}}^{\mathrm{TRD}}$ is the fastest.
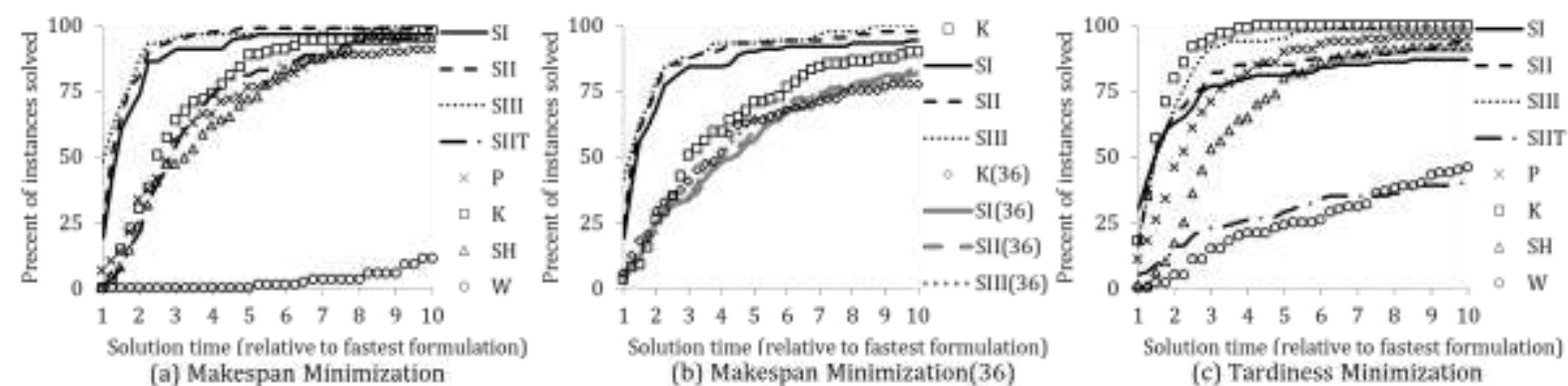

Figure 10. Performance charts of different models for single unit instances using C1 set of options. Note. Models with the same objective but different changeover constraints are compared in each sub-plot: (a) makespan minimization; (b) makespan minimization with valid inequalities (36); and (c) tardiness minimization. The changeover constraints are shown in the legends. 
All models have the same number of variables. Figure 11, in logarithmic scale, summarizes the data for the number of constraints, nonzeros, and nodes in the branch-and-bound tree. Detailed model and solution statistics are given in Supplementary Material. The models with pairwise changeover constraints, $\mathrm{M}_{\mathrm{P}}, \mathrm{M}_{\mathrm{SH}}, \mathrm{M}_{\mathrm{W}}$, have about an order of magnitude more constraints than the models with single-task constraints, potentially explaining why they are slower. The tightest models, $\mathrm{M}_{\mathrm{W}}$ and $\mathrm{M}_{\mathrm{SIIT}}$, have the most non-zeros, the number of which is more than 5 times greater than that of the fastest model $\mathrm{M}_{\mathrm{SIII}}$. The changeover constraints account for more than $99 \%$ of the total constraints for models with pairwise changeover constraints, and for $91 \%-95 \%$ of the constraints for the models with single-task constraints. With CPLEX setting $\mathrm{C} 1$, we find that $\mathrm{M}_{\mathrm{SI}}$ leads to the smallest branch-and-bound tree, while $\mathrm{M}_{\mathrm{K}}$ being the least tight model has the largest tree, with an order of magnitude more nodes searched compared to $\mathrm{M}_{\mathrm{SI}}$. If we also turn off the cut generation process in CPLEX, then the number of nodes increases for all models; more importantly, we find that the number of nodes for other models is more than 5 times larger than that for $\mathrm{M}_{\text {SIIT }}$, which is expected from the theoretical tightness.

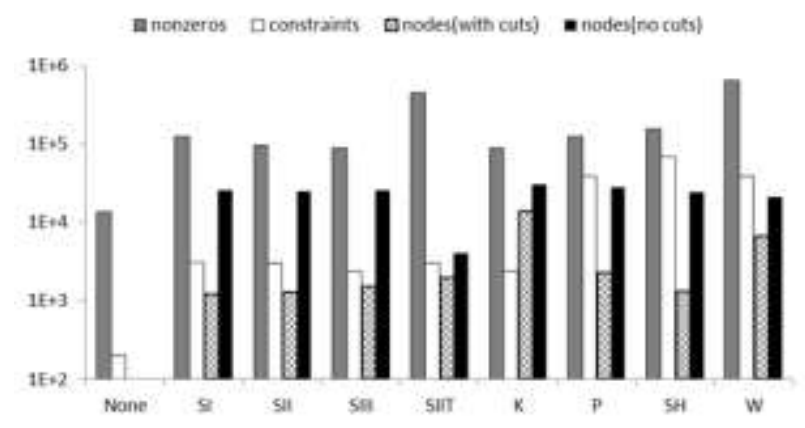

Figure 11. Average over all single unit instances of the number of constraints, nonzeros, and branch-andbound nodes. None refers to the model without changeovers.

Table 4 presents the average improvement of the integrality gap with respect to the gap of $M_{K}$, which has the largest gap (the integrality gaps of any two models can be compared using the relative gap with respect to $M_{K}$ ). Based on these results, models $M_{\text {SIIT }}$ and $M_{W}$ have the greatest integrality gap improvement, followed by $\mathrm{M}_{\mathrm{SI}}, \mathrm{M}_{\mathrm{SII}}, \mathrm{M}_{\mathrm{P}}, \mathrm{M}_{\mathrm{SIII}}$, and then by $\mathrm{M}_{\mathrm{SH}}$, an observation that is in agreement with the theoretical study in the previous section. Note that the gap for tardiness minimization varies across changeover constraints much more than that for makespan minimization.

Table 4. Single-unit problem: average integrality gap improvement with respect to the gap of $\mathrm{M}_{\mathrm{K}}$.

\begin{tabular}{ccccccccc}
\hline Problem & K (ref) & SI & SII & SIII & SIIT & P & SH & W \\
\hline Makespan & 0.94 & $0 \%$ & $0 \%$ & $0 \%$ & $0.04 \%$ & $0 \%$ & $0 \%$ & $0 \%$ \\
Tardiness & 0.28 & $12.10 \%$ & $12.0 \%$ & $10.42 \%$ & $36.14 \%$ & $11.24 \%$ & $9.90 \%$ & $33.76 \%$ \\
\hline
\end{tabular}

\subsection{Parallel Units}

The time horizon was selected based on the number of tasks, the number of units and the longest processing time as follows, 


$$
\eta=\left(\max _{i, j} \tau_{i j}\right) \cdot\left\lfloor\frac{2|\mathbf{I}|-1}{|\mathbf{J}|}\right\rfloor
$$

The term in the round-down operator is the maximum number of tasks plus changeovers that we would expect to take place in a single unit; note that we only need the horizon to be long enough to start the last task, so we rounded down. We multiplied this term by the maximum processing time of any task.

Figure 12 shows performance charts for makespan minimization with and without valid inequalities (36), for tardiness minimization, and for cost minimization. As in the single-unit problems (§6.1), we see that the models with single-task changeover constraints are faster. $\mathrm{M}_{\mathrm{SII}}^{\mathrm{MS}}$ is the fastest among $\mathrm{M}^{\mathrm{MS}}$. We tested the models with the four fastest changeover constraints together with valid inequalities (36), and see improvements as expected. With valid inequalities (36), $\mathrm{M}_{\mathrm{SII}}^{\mathrm{MS}-\mathrm{VI}}, \mathrm{M}_{\mathrm{SIII}}^{\mathrm{MS}-\mathrm{VI}}, \mathrm{M}_{\mathrm{K}}^{\mathrm{MS}-\mathrm{VI}}$ are the fastest among $\mathrm{M}^{\mathrm{MS}-\mathrm{VI}}$, and they are faster than $\mathrm{M}^{\mathrm{MS}}$. For tardiness minimization, $\mathrm{M}_{\mathrm{K}}^{\mathrm{TRD}}$ is the fastest; while for cost minimization, $\mathrm{M}_{\mathrm{SII}}^{\mathrm{CST}}$ and $\mathrm{M}_{\mathrm{SIII}}^{\mathrm{CST}}$ are the fastest. Table 5 shows the average integrality gap improvement for different models with respect to $M_{K}$. Similar to the results in $\S 6.1, \mathrm{M}_{\mathrm{SIIT}}$, has the greatest integrality gap reduction.

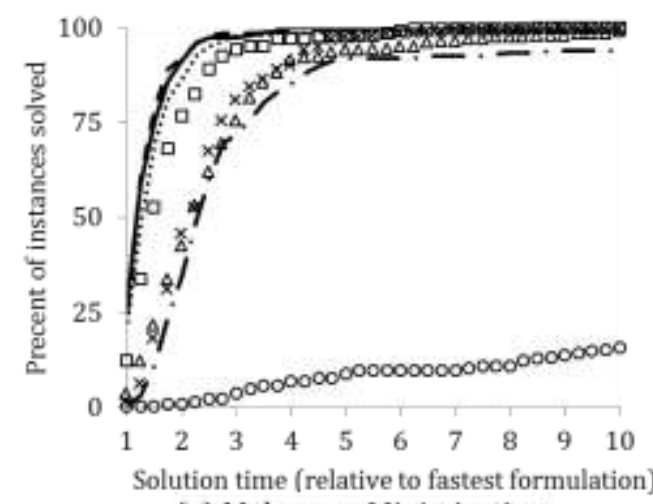

(a) Makespan Minimization

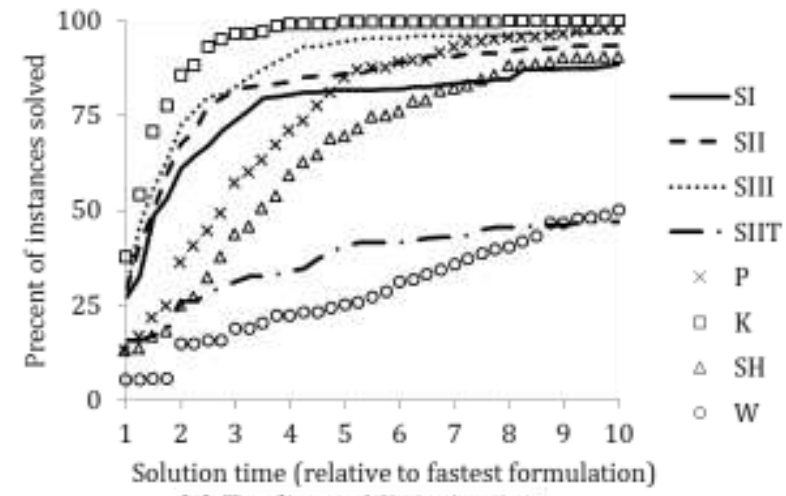

(c) Tardiness Minimization

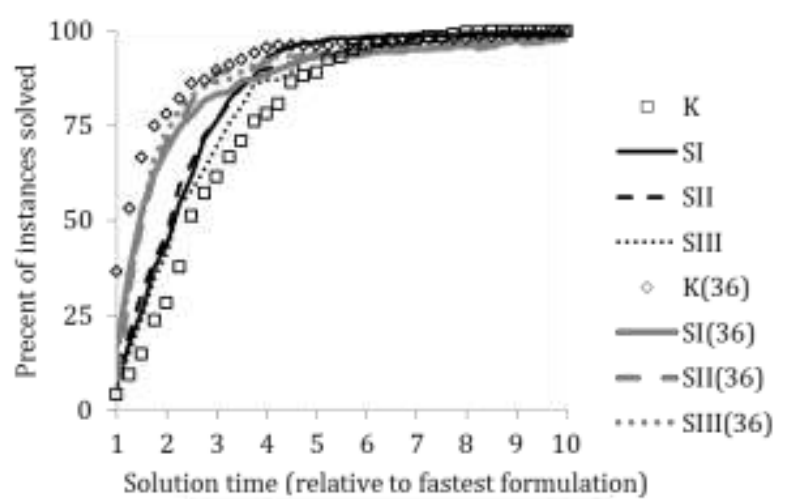

(b) Makespan Minimization(36)

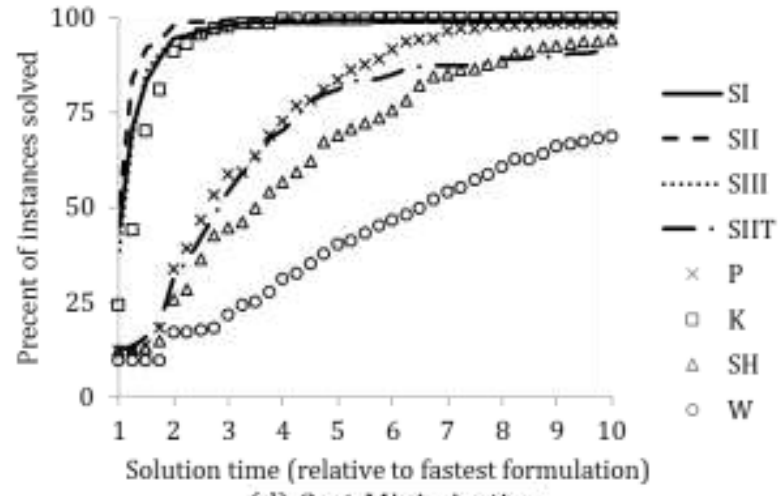

(d) Cost Minimization

Figure 12. Performance charts of different models for instances with parallel units using $\mathrm{C} 1$ set of options. 
Table 5. Parallel units problem: average integrality gap improvement with respect to the gap of $\mathrm{M}_{\mathrm{K}}$.

\begin{tabular}{ccccccccc}
\hline Problem & K(ref) & SI & SII & SIII & SIIT & P & SH & W \\
\hline Makespan & 0.91 & $0 \%$ & $0 \%$ & $0 \%$ & $0.06 \%$ & $0 \%$ & $0 \%$ & $0.03 \%$ \\
Tardiness & 0.10 & $12.03 \%$ & $12.01 \%$ & $11.23 \%$ & $24.77 \%$ & $11.53 \%$ & $10.32 \%$ & $19.89 \%$ \\
Cost & 0.04 & $29.78 \%$ & $29.78 \%$ & $29.78 \%$ & $36.72 \%$ & $29.51 \%$ & $29.13 \%$ & $36.42 \%$ \\
\hline
\end{tabular}

\subsection{Parallel Units with Unequal Capacities}

Unit capacities were randomly selected with a uniform distribution from 10-30 kg, and customer demands were randomly selected from $20-40 \mathrm{~kg}$. The time horizon for the makespan minimization instances was chosen as

$$
\eta=\left\{\max _{i, j}\left[\tau_{i j}\left[\xi_{i} / \beta_{j}\right]\right]\right\} \cdot\left\lfloor\frac{2|\mathbf{I}|-1}{|\mathbf{J}|}\right\rfloor
$$

The first term is the maximum total time any task needs to run for to meet demand, which we found by multiplying the processing time by the number of runs required in each unit and taking the maximum over all units. The second term is the number of tasks plus changeovers expected on a single unit.

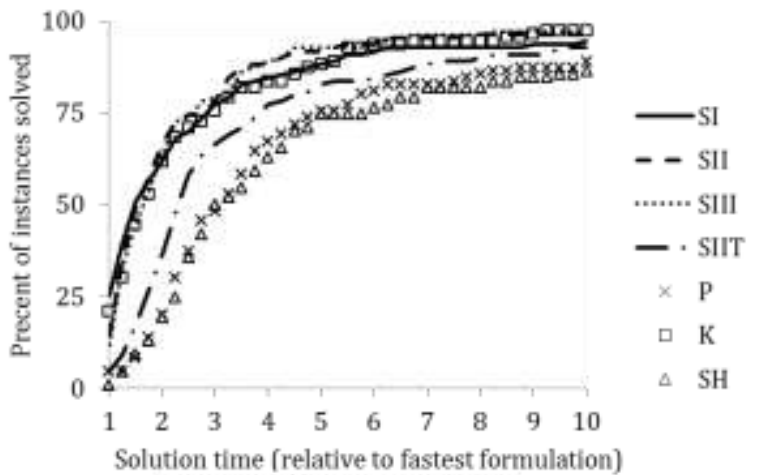

(a) Makespan Minimization

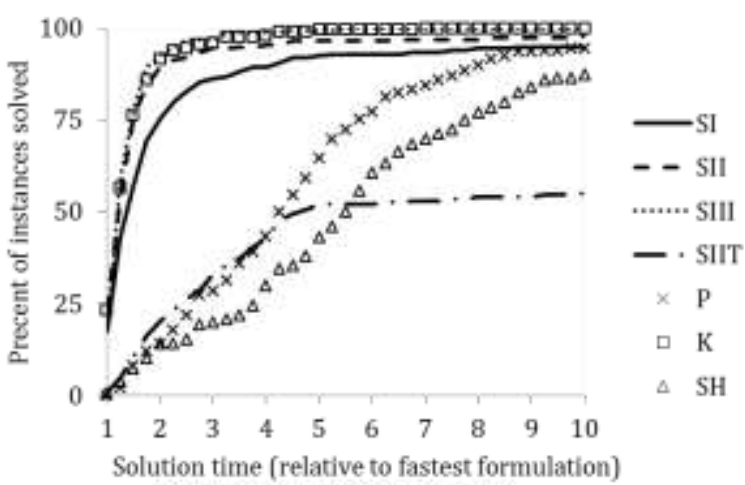

(c) Tardiness Minimization

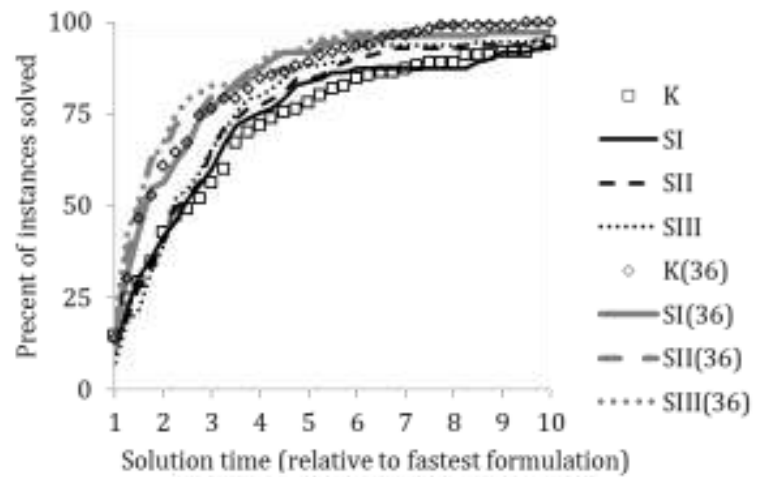

(b) Makespan Minimization(36)

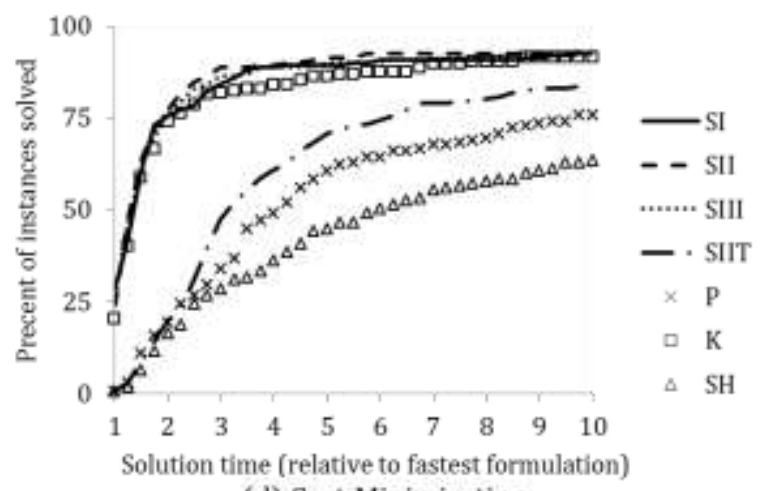

(d) Cost Minimization

Figure 13. Performance charts of different models for unequal capacity parallel units instances using C1.

Figure 13 shows the performance charts. Constraints (W) are not tested because they are not applicable when a task can be executed multiple times. For makespan minimization, $\mathrm{M}_{\mathrm{SII}}^{\mathrm{MS}}, \mathrm{M}_{\mathrm{SIII}}^{\mathrm{MS}}$, $\mathrm{M}_{\mathrm{K}}^{\mathrm{MS}}$ are the fastest, while $\mathrm{M}_{\mathrm{SIIT}}^{\mathrm{MS}}$ and the two models with pairwise changeover constraints $\mathrm{M}_{\mathrm{P}}^{\mathrm{MS}}$ and $\mathrm{M}_{\mathrm{SH}}^{\mathrm{MS}}$ are the slowest. Same as in the parallel units problems (§6.2), adding valid inequalites (36) 
significantly improves the solution times for makespan minimization. For tardiness minimization, $\mathrm{M}_{\mathrm{SIII}}^{\mathrm{TRD}}$ and $\mathrm{M}_{\mathrm{K}}^{\mathrm{TRD}}$ are the fastest, while for cost minimization, $\mathrm{M}_{\mathrm{SII}}^{\mathrm{CST}}$ and $\mathrm{M}_{\text {SIII }}^{\mathrm{CST}}$ are the most fastest. Table 6 compares the improvement of integrality gaps of the models.

Table 6. Parallel units with unequal capacities: average integrality gap improvement with respect to $M_{K}$.

\begin{tabular}{cccccccc}
\hline Problem & K(ref) & SI & SII & SIII & SIIT & P & SH \\
\hline Makespan & 0.94 & $0 \%$ & $0 \%$ & $0 \%$ & $0.04 \%$ & $0 \%$ & $0 \%$ \\
Tardiness & 0.35 & $0.94 \%$ & $0.94 \%$ & $0.87 \%$ & $2.69 \%$ & $0.82 \%$ & $0.80 \%$ \\
Cost & 0.26 & $3.43 \%$ & $3.43 \%$ & $3.21 \%$ & $5.18 \%$ & $3.19 \%$ & $3.10 \%$ \\
\hline
\end{tabular}

\subsection{Additional Testing}

We tested the constraints with additional binary variables included on the LHS (constraints (27)(31)). Also, since the changeover constraints make up a significant portion of the total constraints (Figure 11), we used the lazy constraints feature in CPLEX (IBM ILOG, 2015). Lazy constraints are initially excluded but are automatically added if violated. They can be added by modifying the LP files or using callbacks (we used the former approach). Typically less than $10 \%$ of the total changeover constraints were added. We tested the models with the tightened formulations (constraints (27)-(31)), and the original formulation with the lazy constraints feature on the singleunit problem. Figure 14 compares solution times using the original formulation, the tightened formulation (without the lazy constraints feature), and the original formulation with the lazy constraints feature (without tightening). Using the tightened formulation increases the average solution time by 5\%-205\%; while using lazy constraints increases the average solution time by $4 \%$ $201 \%$.

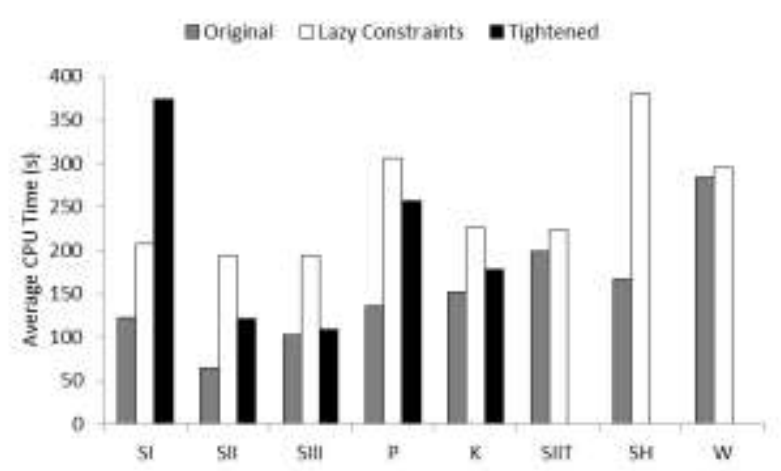

Figure 14. Average solution time of single-unit instances for the original formulations, the original formulations with the lazy constraints feature, and the tightened formulations.

We also studied the performance of all models using CPLEX default settings (C2). The relative performance of the different changeover constraints remains the same, though solution times are shorter (detailed results are given in Supplementary Material). Table 7 summarizes how valid inequalities (36) affect the solution time for makespan minimization problems, using C1 or C2. For the single unit problem, the average solution time using valid inequalities (36) and C1 is 1\%-106\% higher, though it decreases by 36\%-48\% when C2 settings are used. For the parallel units problem, the average solution time using valid inequalities (36) decreases by $1 \%-29 \%$ when $\mathrm{C} 1$ is used and by $39 \%-48 \%$ when C2 is used. For the instances with unequal capacity units, solution times using 
valid inequalities (36) decrease by 18\%-33\% (10\%-13\%) when using C1 (C2). We also tested all instances with both preprocessing and cut generation turned off. The computational times in this case increase significantly: only $63 \%$ of the instances were solved to optimality within 3600 seconds. Finally, we want to mention that a continuous-time model was also tested (see Supplementary Material).

Table 7. Solution times for makespan minimization problems (different formulations and valid inequalities).

\begin{tabular}{cccccccccc}
\hline Problem & CPLEX & $\mathrm{K}$ & SI & SII & SIII & $\mathrm{K}(36)$ & SI(36) & SII(36) & SIII(36) \\
\hline \multirow{2}{*}{ Single unit } & C1 & 1.00 & 0.49 & 0.48 & 0.57 & 1.01 & 1.01 & 0.96 & 0.93 \\
& C2 & 0.31 & 0.33 & 0.32 & 0.34 & 0.20 & 0.17 & 0.16 & 0.19 \\
Parallel units & C1 & 1.00 & 0.94 & 0.94 & 0.99 & 0.71 & 0.93 & 0.93 & 0.96 \\
Parallel units with & C2 & 0.55 & 0.79 & 0.77 & 0.73 & 0.34 & 0.41 & 0.41 & 0.40 \\
unequal capacities & C2 & 1.00 & 1.12 & 1.00 & 0.99 & 0.82 & 0.75 & 0.73 & 0.71 \\
\hline
\end{tabular}

Note. The solution time of each instance is normalized with respect to the solution time for $\mathrm{M}_{\mathrm{K}}^{\mathrm{MS}}$ using CPLEX setting C1. We use the average normalized computational time over all instances for the same type of problem.

\section{Conclusions}

In this paper, we presented five new formulations for sequence-dependent changeover times in discrete-time MIP scheduling models, and compared them with previous formulations both theoretically and computationally. The proposed constraints are written either for a single task or for pairs of tasks. In terms of tightness, four of the new changeover constraints, (SI), (SII), (SIIT) and $(\mathrm{P})$, are tighter than the two literature constraints, $(\mathrm{SH})$ and $(\mathrm{K})$. Among the constraints written for a single task, constraints (K) are the weakest, followed by (SIII) and (SII); and (SI) and (SIIT) are the tightest. Among the constraints written for pairs of tasks, constraints (W) are the tightest, and (SH) are the weakest. Constraints (W), however, cannot be applied to problems in which a task can be executed more than once. In terms of computational effectiveness using CPLEX, although models with constraints (K), (SIII), (SII), and (SI) have similar solution times, models with constraints (SII) and (SIII) are typically the fastest. We observed that tighter formulations did not necessarily lead to faster computational times, as tighter formulations had typically more constraints and/or nonzeros, and their LP relaxations were computationally more expensive (see Supplementary Material). Also, models with changeover constraints written for a single task were faster than those written for pairs of tasks. We observe similar computational results using Gurobi (see Supplementary Material).

The proposed constraints can be added to any discrete-time MIP scheduling formulation, including models developed to address problems in complex production environments (e.g., environments with batch mixing, splitting and recycling) as well as problems with a range of processing characteristics (e.g., general resource constraints, time-varying resource availability and cost). Thus, the constraints presented herein are relevant for many real-world problems. 


\section{Acknowledgements}

The authors would like to acknowledge financial support from the National Science Foundation under grant CBET-1066206 and Petroleum Research Fund under grand 53313-ND9.

\section{Notation}

\section{Indices/ Sets}

$i \in \mathbf{I} \quad$ Tasks (jobs)

$j \in \mathbf{J} \quad$ Units (machines)

$t \in \mathbf{T} \quad$ Time points/periods

$n \in \mathbf{N} \quad$ Index used to select times points when a constraint is written

\section{Subsets}

$\mathbf{I}_{j} \quad$ Tasks that can be processed in unit j

$\mathbf{J}_{i} \quad$ Units that can process task $i$

$\mathbf{N}_{i j} \quad$ Indices $\mathrm{n}$ for which $v_{i j n}$ and $\mu_{i j n}$ are defined

$\mathbf{T}_{i j t}^{P} \quad=\left\{t^{\prime} \mid t-\tau_{i j}+1 \leq t^{\prime} \leq t\right\}$, for given $(i, j, t)$

$\mathbf{T}_{i^{\prime} i j t}^{C} \quad=\left\{t^{\prime} \mid t-\tau_{i^{\prime}{ }^{\prime}}-\sigma_{i^{\prime}}{ }^{\prime}{ }^{\prime}+1 \leq t^{\prime} \leq t-\tau_{i^{\prime} j}\right\}$, for given $\left(i, i^{\prime}, j, t\right)$

\section{Parameters}

$\alpha_{i j} \quad$ Cost to run task $i$ in unit j

$\beta_{j} \quad$ Capacity of unit j

$\delta \quad$ Time step

$\varepsilon \quad$ Factor used to determine the maximum changeover length

$\eta \quad$ Time horizon

$v_{i j n} / \mu_{i j n}$ Parameter to select the time points for which (SII)/(SIIT) is written for task $i$ and unit $\mathrm{j}$

$\xi_{j} \quad$ Demand for the output of task $i$

$\sigma_{i i}{ }^{\prime} j \quad$ Changeover time between task $i$ and task $i^{\prime}$ on unit j

$\tau_{i j} \quad$ Fixed processing time for task $i$ in unit $j$

$\phi_{i} / \bar{\phi}_{i} \quad$ Due time/dealine of task $i$

$\omega a_{i j n i^{\prime}} / \omega b_{i j n i^{\prime}}$ Parameters to define the summation over $i^{\prime}$ for given $(i, j, n)$ in (SIIT)

\section{Binary Variables}

$X_{i j t}=1$ if and only if task $i$ starts on unit $\mathrm{j}$ at time point $\mathrm{t}$

\section{Continuous Nonnegative Variables}

CST Total cost

MS Makespan

$T R D_{i} \quad$ Tardiness for task $i$

TRD Total tardiness 


\section{Appendix 1: Proof of correctness of constraints (SI)}

Since the changeover constraints for different units are independent, without loss of generality we drop index $j$. Thus, constraints (SI) become

$$
X_{i t}+\sum_{i^{\prime} \neq i t^{\prime \prime}=\max } \sum_{\left\{t^{\prime}, t-\sigma_{i^{\prime}}\right\}-\tau_{i^{\prime}}+1}^{\min \left\{t^{\prime}, t-\tau_{i^{\prime}}\right\}} X_{i^{\prime}} t^{\prime \prime} \leq 1 \quad \forall i, t, t-\max \left\{\min _{i \neq i} \tau_{i^{\prime}}, \max _{i^{\prime} \neq i} \sigma_{i^{\prime} i}\right\} \leq t^{\prime} \leq t-\min _{i \neq i} \tau_{i^{\prime}}
$$

To prove the correctness of (SI), we need to show two things for given $(i, t)$ : (1) (SI) include all variables corresponding to $t^{\prime} \in \mathbf{T}_{i^{\prime}}^{C}$ it for each task $i^{\prime} \neq i$; and (2) no valid solutions are cut off.

To prove the first part, we show that all $X_{i^{\prime}} t^{\prime}$ for any $i^{\prime} \neq i$ and $t^{\prime} \in\left\{t-\tau_{i^{\prime}}-\sigma_{i^{\prime} i}+1, \ldots, t-\tau_{i}{ }^{\prime}\right\}$ are included in at least one constraint. For every $i^{\prime} \neq i$, there are three cases:

(1) When $t^{\prime}=t-\min _{i}{ }^{\prime \prime} \neq i\left\{\tau_{i^{\prime \prime}}\right\}$, the variable $X_{i^{\prime}} t^{\prime \prime}$ with $t^{\prime \prime}=t-\tau_{i}{ }^{\prime}$, which is the last changeover time point, is included in the constraint.

(2) When $t^{\prime} \leq t-\sigma_{i^{\prime}}{ }_{i}$, the variable $X_{i^{\prime}} t^{\prime \prime}$ with $t^{\prime \prime}=t-\tau_{i}{ }^{\prime}-\sigma_{i^{\prime} i}+1$, which is the first changeover time point, is included in the constraint.

(3) When $t^{\prime}$ decreases by one, the largest time index $t^{\prime \prime}$ of the variables in the summation stays the same or decreases by one, which means that all variables with time index between the first and the last changeover time point are also included.

To prove that no valid solutions are cut off, we show that no feasible assignments are excluded:

(1) For each $i^{\prime}$, the variables included in one inequality span at most $\tau_{i}{ }^{\prime}$ time points; thus, no solution with the same task scheduled back-to-back is cut off.

(2) The smallest time index of the included $X_{i}{ }^{\prime} t^{\prime \prime}$ variables is $t^{\prime \prime}=t-\tau_{i}{ }^{\prime}-\sigma_{i}{ }^{\prime}{ }+1$. Thus, no solution with task $i$ scheduled after $i^{\prime}$ is cut off.

(3) For any $i_{1} \neq i$ and $i_{2} \neq i$, the smallest index of included $X_{i_{1} t^{\prime \prime}}$ is $t_{1}^{L}=\max \left\{t^{\prime}, t-\sigma_{i 1, i}\right\}-\tau_{i 1}+$ $1 \geq t^{\prime}-\tau_{i 1}+1$, and the largest index of included $X_{i_{2}} t^{\prime \prime}$ is $t_{2}^{U}=\min \left\{t^{\prime}, t-\tau_{i 2}\right\} \leq t^{\prime}$. Since $t_{2}^{U}-t_{1}^{L} \leq \tau_{i 1}-1<\tau_{i 1}+\sigma_{i 1, i 2}$, no solution with task $i_{2}$ scheduled after $i_{1}$ is cut off.

\section{Appendix 2: Proof of correctness of constraints (SIIT)}

Since the changeover constraints for different units are independent, we drop index $j$ without loss of generality. Thus, constraints (SIIT) become

$$
\sum_{t^{\prime}=t-\tau_{i}+1}^{t} X_{i t^{\prime}}+\sum_{i \neq i} \sum_{t^{\prime}=t-\omega a_{i n i^{\prime}}}^{t-\omega b_{i n i^{\prime}}} X_{i^{\prime}} t^{\prime} \leq 1 \quad \forall i, t, n \in \mathbf{N}_{i}
$$

To prove the correctness of (SIIT) for given $(i, t)$, we need to show two things: (SIIT) forces all variables corresponding to $i^{\prime} \neq i$ and $t^{\prime} \in \mathbf{T}_{i^{\prime}}^{C}$ it to be zero if $X_{i t}=1$, and no valid solutions are cut off.

First, we show that for any $i^{\prime} \neq i$ and $t^{\prime} \in\left\{t-\tau_{i^{\prime}}-\sigma_{i^{\prime} i}+1, \ldots, t-\tau_{i^{\prime}}\right\}, X_{i^{\prime}} t^{\prime}$ is included in at least one constraint of $n \in \mathbf{N}_{i}$, based on the following three observations. For all $i^{\prime} \neq i$, we have: 
(1) $X_{i^{\prime} t^{\prime}}$ for $t^{\prime}=t-\tau_{i}{ }^{\prime}$, which is the largest $t^{\prime} \in \mathbf{T}_{i^{\prime}}^{C}$, , is included in the constraint for $n=1$ since $\omega b_{i 1 i^{\prime}} \leq \tau_{i^{\prime}} \leq \omega a_{i 1 i^{\prime}}$ for either $i^{\prime} \in \mathbf{I} \mathbf{A}_{\text {in }}$ or $i^{\prime} \in \mathbf{I B}_{i n}$.

(2) $X_{i^{\prime}} t^{\prime}$ for $t^{\prime}=t-\tau_{i^{\prime}}-\sigma_{i^{\prime} i}+1$, which is the first changeover time point, is included in the constraint for the smallest element in $\mathbf{N}_{i}$ for which $\mu_{i n} \geq \sigma_{i^{\prime}}{ }^{\prime}$ (denoted by $n_{i}{ }^{\prime}$ ).

(3) When $n$ is less than $n_{i}{ }^{\prime}$, we have $\omega b_{i, n+1, i^{\prime}} \leq \mu_{i, n+1} \leq \mu_{i n}+\tau_{i}{ }^{\prime}=\omega a_{\text {in } i^{\prime}}+1$, which means that variables $X_{i^{\prime}}{ }^{\prime}{ }^{\prime}$ for all changeover times (i.e., $t^{\prime} \in \mathbf{T}_{i^{\prime} i t}^{C}$ ) are included in at least one inequality.

Second, to prove that no valid solutions are cut off, we consider the following cases for given $(i, t)$ :

(1) The same task scheduled back-to-back. For $i$ or $i^{\prime}$, the time indices of the binary variables included in one of the inequalities have a difference less than or equal to the processing time of this task, thus, no solution with the same task scheduled back-to-back is cut off.

(2) A task $i^{\prime} \neq i$ scheduled after $i$. The smallest time index of the included $X_{i t^{\prime}}$ variables is $t_{1}^{L}=t-\tau_{i}+1$, and the largest time index of the included $X_{i^{\prime}} t^{\prime}$ variables cannot be larger than $t_{2}^{U}=t$. Since $t_{2}^{U}-t_{1}^{L}=\tau_{i}-1<\tau_{i}+\sigma_{i i^{\prime}}$, no solution with $i^{\prime}$ scheduled after $i$ is cut off.

(3) A task $i^{\prime} \neq i$ is scheduled before $i$. The smallest time index of the included $X_{i^{\prime}} t^{\prime}$ variables is $t_{1}^{L}=t-\tau_{i}{ }^{\prime}-\min \left\{\sigma_{i}{ }^{\prime}{ }_{i}, \mu_{i n}\right\}+1 \geq t-\tau_{i}{ }^{\prime}-\sigma_{i^{\prime}}{ }+1$, and the largest time index of the included $X_{i t^{\prime}}$ variables is $t_{2}^{U}=t$. Once again $t_{2}^{U}-t_{1}^{L} \leq \tau_{i^{\prime}}+\sigma_{i^{\prime} i}-1$, so no solution with task $i$ scheduled after $i^{\prime}$ is cut off.

(4) Two tasks, both in $\mathbf{I} \mathbf{A}_{\text {in }}$, are scheduled back-to-back. The included variables have the same largest time index, $t-\mu_{\text {in }}$. Also, the difference between the largest and the smallest $t^{\prime}$ of the included $X_{i^{\prime}} t^{\prime}$ variables, is $\tau_{i^{\prime}}-1$. Thus, no solution with these tasks scheduled one after another is cut off.

(5) Two tasks $i_{1}, i_{2}$, both in $\mathbf{I B}_{\text {in }}$, are scheduled back-to-back. The smallest time index of the included $X_{i_{1} t^{\prime}}$ variables is $t_{1}^{L}=t-\tau_{i 1}-\sigma_{i 1, i}+1$, while the time index of the included $X_{i_{2} t^{\prime}}$ variables cannot be larger than $t_{2}^{U}=t-\sigma_{i 1, i}+\sigma_{i 1, i 2}$. Since $t_{2}^{U}-t_{1}^{L}=\tau_{i 1}+\sigma_{i 1, i 2}-1$, no solution with task $i_{2}$ scheduled after $i_{1}$ is cut off.

(6) Task $i_{1} \in \mathbf{I} \mathbf{A}_{i n}$ is scheduled before $i_{2} \in \mathbf{I B}_{\text {in }}$. The smallest time index of the included $X_{i_{1}} t^{\prime}$ variables is $t_{1}^{L}=t-\mu_{i n}-\tau_{i 1}+1$, while the time index of $X_{i_{2}} t^{\prime}$ variables cannot be larger than $t_{2}^{U}=t-\mu_{i n}+\sigma_{i 1, i 2}$. Similar to (5), no solution with task $i_{1}$ scheduled before $i_{2}$ is cut off.

(7) Task $i_{1} \in \mathbf{I} \mathbf{A}_{\text {in }}$ is scheduled after $i_{2} \in \mathbf{I B}_{\text {in }}$. The smallest time index of the included $X_{i_{2}} t^{\prime}$ variables is $t_{2}^{L}=t-\tau_{i 2}-\sigma_{i 2, i}+1$, while the time index of $X_{i_{1} t^{\prime}}$ variables cannot be larger than $t_{1}^{U}=t-\mu_{i n}$. The difference is $\tau_{i 2}+\sigma_{i 2, i}-\mu_{i n}-1$. Since $\mu_{i n}>\sigma_{i 2, i}\left(i_{2} \in \mathbf{I B}_{i n}\right)$, no solution with task $i_{1}$ scheduled after $i_{2}$ is cut off.

\section{Appendix 3: Proof of Theorem 1}

Since changeovers in different units are independent, index $j$ is dropped without loss of generality.

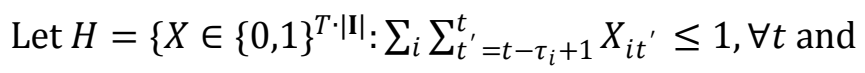

$\left.\sum_{t^{\prime}=t-\tau_{i}+1}^{t} X_{i t^{\prime}}+\sum_{i^{\prime} \neq i} \sum_{t^{\prime}=t-\omega a_{i n i^{\prime}}^{t-\omega b_{i n}}}^{\prime^{\prime}} X_{i^{\prime} t^{\prime}} \leq 1, \forall i, t, n \in \mathbf{N}_{i}\right\}$. 
We want to prove that each face $F_{i t n}$ defined by (A3) is facet-defining for the convex hull, $\operatorname{conv}(\mathrm{H})$. We will prove it by showing that the face defined in expression (A3) has dimension $\mathrm{T} \cdot|\mathbf{I}|-1$, which is one less than the dimension of $\operatorname{conv}(\mathrm{H})$, as there are $\mathrm{T} \cdot|\mathbf{I}| X_{i t}$ variables and $\mathrm{H}$ is full dimensional.

$$
F_{i t n}=\left\{X_{i t} \in \operatorname{conv}(H): \sum_{t^{\prime}=t-\tau_{i}+1}^{t} X_{i t^{\prime}}+\sum_{i \neq i} \sum_{t^{\prime}=t-\omega a_{i n i^{\prime}}}^{t-\omega b_{i n i^{\prime}}} X_{i^{\prime} t^{\prime}}=1\right\} \forall i, t, n \in \mathbf{N}_{i}
$$

To show that $F_{i t n}$ has dimension $\mathrm{T} \cdot|\mathbf{I}|-1$, it suffices to show that it has $\mathrm{T} \cdot|\mathbf{I}|$ affinely independent points.

For given $(i, t, n)$, the following points belong to $F_{i t n}$, and are affinely independent (one point is shown in each curly bracket, and for simplicity of presentation, we only list the non-zero variables):

(1) $\left\{X_{i t^{\prime}}=1\right\}, t^{\prime} \in\left\{t-\tau_{i}+1, \ldots, t\right\}$. These are $\tau_{i}$ points.

(2) $\left\{X_{i t^{\prime}}=1, X_{i, t^{\prime}+k \cdot \tau_{i}}=1\right\}, t^{\prime} \in\left\{1, \ldots, t-\tau_{i}\right\} \cup\{t+1, \ldots, T\}, k \in \mathbb{Z} \backslash\{0\}$ and $t^{\prime}+k \cdot \tau_{i} \in\left\{t-\tau_{i}+\right.$ $1, \ldots, t\}$. Note that $k$, as well as the $k$ listed below, is negative if $t^{\prime}>t$. These are $T-\tau_{i}$ points.

(3) For $i^{\prime} \in \mathbf{I A}_{\text {in }},\left\{X_{i^{\prime}} t^{\prime}=1\right\}, t^{\prime} \in\left\{t-\omega a_{\text {in } i^{\prime}}, \ldots, t-\omega b_{\text {ini }}{ }^{\prime}\right\}$.

(4) For $i^{\prime} \in \mathbf{I A}_{\text {in }}, \quad\left\{X_{i^{\prime} t^{\prime}}=1, X_{i^{\prime}, t^{\prime}+k \cdot \tau_{i}}=1\right\}, t^{\prime} \in\left\{1, \ldots, t-\omega a_{\text {ini }}{ }^{\prime}-1\right\} \cup\left\{t-\omega b_{\text {ini }}{ }^{\prime}+1, \ldots, T\right\}, k \in$ $\mathbb{Z} \backslash\{0\}$ and $t^{\prime}+k \cdot \tau_{i} \in\left\{t-\omega a_{i n i^{\prime}}, \ldots, t-\omega b_{i n i^{\prime}}\right\}$. Cases (3) and (4) have $T \cdot\left|\mathbf{I} \mathbf{A}_{\text {in }}\right|$ points.

(5) For $i^{\prime} \in \mathbf{I B}_{\text {in }},\left\{X_{i^{\prime}} t^{\prime}=1\right\}, t^{\prime} \in\left\{t-\omega a_{\text {ini }}{ }^{\prime}, \ldots, t-\omega b_{i n i^{\prime}}\right\}$.

(6) For $i^{\prime} \in \mathbf{I B}_{i n},\left\{X_{i^{\prime}} t^{\prime}=1, X_{i t}=1\right\}, t^{\prime} \in\left\{1, \ldots, t-\omega a_{i n i^{\prime}}-1\right\}$.

(7) For $i^{\prime} \in \mathbf{I B}_{\text {in }}$ and $t^{\prime} \in\left\{t-\omega b_{i n i^{\prime}}+1, \ldots, T\right\}$, based on the definition of $\omega b_{\text {ini }}{ }^{\prime}$, at least one of the following three scenarios is true, and we can select the point accordingly (in case when more than one scenario is true, we select one of them). Cases (5) - (7) have $T \cdot\left|\mathbf{I B}_{\text {in }}\right|$ points.

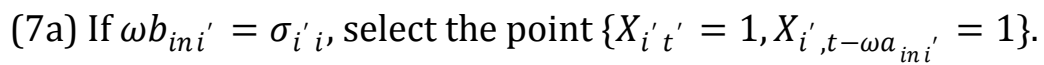

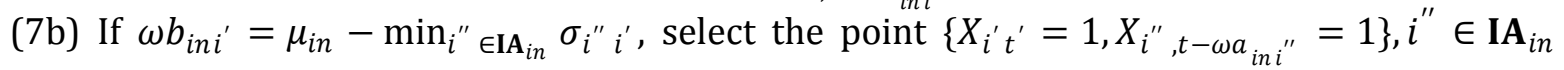
and $\sigma_{i}^{\prime \prime} i^{\prime}=\min _{i}^{\prime \prime \prime} \in \mathbf{I} \mathbf{A}_{i n} \sigma_{i}^{\prime \prime \prime} i^{\prime}$.

(7c) If $\omega b_{i n i^{\prime}}=\max _{i^{\prime \prime} \in \mathbf{I B}_{i n} \backslash\left\{i^{\prime}\right\}}\left\{\sigma_{i^{\prime \prime}}{ }_{i}-\sigma_{i^{\prime \prime}} i^{\prime}\right\}$, select the point $\left\{X_{i^{\prime} t^{\prime}}=1, X_{i^{\prime \prime}, t-\omega a_{i n} i^{\prime \prime}}=1\right\}, i^{\prime \prime} \in$ $\mathbf{I B}_{\text {in }}$ and $\sigma_{i^{\prime \prime}}{ }_{i}-\sigma_{i^{\prime \prime} i^{\prime}}=\max _{i}^{\prime \prime \prime} \in \mathbf{I B}_{\text {in }} \backslash\left\{i^{\prime}\right\}\left\{\sigma_{i}^{\prime \prime \prime}{ }_{i}-\sigma_{i^{\prime \prime \prime}}{ }^{\prime}\right\}$.

From the set definition, we have $\left|\mathbf{I A}_{\text {in }}\right|+\left|\mathbf{I B}_{\text {in }}\right|+1=|\mathbf{I}|$. Thus, there are totally $\mathrm{T} \cdot|\mathbf{I}|$ points in (1) to (7). It is trivial to show these points are linearly independent, and thus affinely independent.

\section{References}

Artigues, C. and D. Feillet (2008). A branch and bound method for the job-shop problem with sequencedependent setup times. Annals of Operations Research 159(1): 135-159.

Avalos-Rosales, O., A. Alvarez and F. Angel-Bello (2013). A reformulation for the problem of scheduling unrelated parallel machines with sequence and machine dependent setup times. Proceedings of the Twenty-Third International Conference on Automated Planning and Scheduling (ICAPS2013), Rome, Italy, 278-282.

Baptiste, P., L. Peridy and E. Pinson (2003). A branch and bound to minimize the number of late jobs on a single machine with release time constraints. European Journal of Operational Research 144(1): 1-11.

Brucker, P. and O. Thiele (1996). A branch \& bound method for the general-shop problem with sequence dependent setup-times. Or Spektrum 18(3): 145-161.

Chandra, C., Z. X. Liu, J. He and T. Ruohonen (2014). A binary branch and bound algorithm to minimize maximum scheduling cost. Omega-International Journal of Management Science 42(1): 9-15. 
Coban, E. and J. N. Hooker (2013). Single-facility scheduling by logic-based Benders decomposition. Annals of Operations Research 210(1): 245-272.

Harjunkoski, I., C. T. Maravelias, P. Bongers, P. M. Castro, S. Engell, I. E. Grossmann, J. Hooker, C. Méndez, G. Sand and J. Wassick (2014). Scope for industrial applications of production scheduling models and solution methods. Computers \& Chemical Engineering 62: 161-193.

Hooker, J. (2007a). Integrated methods for optimization. New York, Springer.

Hooker, J. N. (2007b). Planning and scheduling by logic-based benders decomposition. Operations Research 55(3): 588-602.

IBM ILOG (2015). IBM ILOG CPLEX Optimization Studio V12.6.3 documentation.

Jain, V. and I. E. Grossmann (2001). Algorithms for hybrid MILP/CP models for a class of optimization problems. INFORMS Journal on Computing 13(4): 258-276.

Karmarkar, U. S. and L. Schrage (1985). The Deterministic Dynamic Product Cycling Problem. Operations Research 33(2): 326-345.

Kelly, J. D. and D. Zyngier (2007). An Improved MILP Modeling of Sequence-Dependent Switchovers for Discrete-Time Scheduling Problems. Industrial \& Engineering Chemistry Research 46(14): 4964-4973.

Kondili, E., C. C. Pantelides and R. W. H. Sargent (1993). A General Algorithm for Short-Term Scheduling of Batch-Operations. 1. Milp Formulation. Computers \& Chemical Engineering 17: 211-227.

Kopanos, G. M., C. A. Méndez and L. Puigjaner (2010). MIP-based decomposition strategies for large-scale scheduling problems in multiproduct multistage batch plants: A benchmark scheduling problem of the pharmaceutical industry. European Journal of Operational Reaserch 207(2): 644-655.

Maravelias, C. T. and I. E. Grossmann (2004). A hybrid MILP/CP decomposition approach for the continous time scheduling of multipurpose batch plants. Computers \& Chemical Engineering 28(10):1921-1949.

Maravelias, C. T. (2006). A decomposition framework for the scheduling of single- and multi-stage processes. Computers \& Chemical Engineering 30(3): 407-420.

Maravelias, C. T. (2012). General framework and modeling approach classification for chemical production scheduling. AIChE Journal 58(6): 1812-1828.

Méndez, C. A., J. Cerda, I. E. Grossmann, I. Harjunkoski and M. Fahl (2006). State-of-the-art review of optimization methods for short-term scheduling of batch processes. Computers \& Chemical Engineering 30(6-7): 913-946.

Merchan, A. F., H. Lee and C. T. Maravelias (2016). Discrete-time mixed-integer programming models and solution methods for production scheduling in multistage facilities. Computers \& Chemical Engineering 94: 387-410.

Moniz, S., A. P. Barbosa-Póvoa and J. P. de Sousa (2013). New General Discrete-Time Scheduling Model for Multipurpose Batch Plants. Industrial \& Engineering Chemistry Research 52(48): 17206-17220.

Novara, F. M., J. M. Novas and G. P. Henning (2016). A novel constraint programming model for large-scale scheduling problems in multiproduct multistage batch plants: Limited resources and campaignbased operation. Computers \& Chemical Engineering 93: 101-117.

Sadykov, R. and L. A. Wolsey (2006). Integer programming and constraint programming in solving a multimachine assignment scheduling problem with deadlines and release dates. INFORMS Journal on Computing 18(2): 209-217.

Sahinidis, N. V. and I. E. Grossmann (1991). MINLP Model for Cyclic Multiproduct Scheduling on Continuous Parallel Lines. Computers \& Chemical Engineering 15(2): 85-103.

Shah, N., C. C. Pantelides and R. W. H. Sargent (1993). A General Algorithm for Short-Term Scheduling of Batch Operations. 2. Computational Issues. Computers \& Chemical Engineering 17(2): 229-244.

Sousa, J. P. and L. A. Wolsey (1992). A Time Indexed Formulation of Non-preemptive Single Machine Scheduling Problems. Mathematical Programming 54(1): 353-367.

Sundaramoorthy, A. and C. T. Maravelias (2011a). Computational Study of Network-Based Mixed-Integer Programming Approaches for Chemical Production Scheduling. Industrial \& Engineering Chemistry Research 50(9): 5023-5040.

Sundaramoorthy, A. and C. T. Maravelias (2011b). A General Framework for Process Scheduling. AIChE Journal 57(3): 695-710.

Tran T.T. and J. C. Beck (2012). Logic-based Benders decomposition for alternative resource scheduling with sequence dependent setups. Proceedings of the Twentieth European Conference on Artificial Intelligence (ECAI2012), Montpellier, France, 774-779. 
van den Akker, J. M., C. A. J. Hurkens and M. W. P. Savelsbergh (2000). Time-indexed formulations for machine scheduling problems: Column generation. INFORMS Journal on Computing 12(2): 111-124.

van den Akker, J. M., C. P. M. van Hoesel and M. W. P. Savelsbergh (1999). A polyhedral approach to singlemachine scheduling problems. Mathematical Programming 85(3): 541-572.

Velez, S. and C. T. Maravelias (2013). Mixed-Integer Programming Model and Tightening Methods for Scheduling in General Chemical Production Environments. Industrial \& Engineering Chemistry Research 52(9): 3407-3423.

Wolsey, L. A. (1990). Valid Inequalities for 0-1 Knapsacks and Mips with Generalised Upper Bound Constraints. Discrete Applied Mathematics 29(2-3): 251-261.

Wolsey, L. A. (1997). MIP modelling of changeovers in production planning and scheduling problems. European Journal of Operational Research 99(1): 154-165.

Wolsey, L. A. (1998). Integer Programming; Wiley: New York, NY.

Zeballos, L. J., J. M. Novas and G. P. Henning (2011). A CP formulation for scheduling multiproduct multistage batch plants. Computers \& Chemical Engineering 35(12): 2973-2989.

Zentner, M. G., J. F. Pekny, G. V. Reklaitis and J. N. D. Gupta (1994). Practical considerations in using modelbased optimization for the scheduling and planning of batch/semicontinuous processes. Journal of Process Control 4(4): 259-280.

Zyngier, D. and J. D. Kelly (2009). Multi-product inventory logistics modeling in the process industries. Optimization and logistics challenges in the enterprise W. Chaovalitwongse, K. C. Furman and P. M. Pardalos. New York, Springer: 61-95. 